\title{
Closed-loop recycling of polyethylene-like materials
}

\author{
Manuel Häußler ${ }^{1 \dagger}$, Marcel Eck ${ }^{1 \dagger}$, Dario Rothauer ${ }^{1}$ and Stefan Mecking ${ }^{1 *}$ \\ ${ }^{1}$ Chair of Chemical Materials Science, University of Konstanz, \\ Department of Chemistry, Universitätsstraße 10, 78457 Konstanz, Germany.
}

* stefan.mecking@uni-konstanz.de, Fax: +49 7531885152

Tel.: +497531882593

$\dagger$ These authors contributed equally to this work.

Plastics are a key component of virtually any technology today. Although their production 10 consumes enormous feedstock resources, plastics are largely disposed of after their useful service life. In terms of a circular economy, ${ }^{1-8}$ desirable re-utilisation of post-consumer sorted polymers ('mechanical recycling') is hampered by deterioration of materials performance. ${ }^{9,10}$ Chemical recycling ${ }^{1,11}$ via depolymerisation to monomer offers an alternative to retain high performance properties. The linear hydrocarbon chains of polyethylene ${ }^{12}$ enable crystalline packing and provide 15 excellent materials properties. ${ }^{13}$ Their inert nature hinders chemical recycling, however, necessitating temperatures $>600{ }^{\circ} \mathrm{C}$ and recovering ethylene with $<10 \%$ yield..$^{3,11,14}$

Here, we show that renewable polycarbonates and polyesters with a low-density of in-chain functional groups as break points in a polyethylene chain can be recycled chemically by solvolysis with $>96 \%$ recovery. At the same time, the break points do not disturb the crystalline polyethylene structure, and the HDPE (high density polyethylene)-like materials properties are fully retained upon recycling. Processing can be performed by common injection moulding and the materials are well-suited for additive manufacturing. Selective removal from model polymer waste streams is possible. The virgin polymers result from polycondensation of long-chain building 
blocks, derived by state-of-the-art catalytic schemes from common plant oil feedstocks, or microalgae oils. ${ }^{15}$ This approach (Extended Data Fig. 1) allows for closed-loop recycling of polyethylene-like materials.

\section{Synthesis and properties of polyethylene-like polycarbonate-18 and polyester-18,18}

5 Commercially available, renewable 1,18-octadecanedioic acid, produced from large-scale biorefining of plant oils by olefin metathesis technology, ${ }^{16-18}$ was employed as a starting material. Esterification and reduction by established techniques afforded the corresponding long-chain $\mathrm{C}_{18}$ dimethylester and $\mathrm{C}_{18}$ diol, respectively. Samples of polyester-18,18 (PE-18,18) and polycarbonate-18 (PC-18) had been prepared previously based on other feedstocks, ${ }^{19,20}$ but the key

10 challenge of achieving polyethylene-like mechanical properties had not been addressed. In our hands the utilisation of diethyl carbonate (DEC) as a co-reagent proved decisive for PC-18 to achieve very high molecular weights (up to $\mathrm{M}_{\mathrm{w}} \sim 300.000 \mathrm{~g} \mathrm{~mol}^{-1}, \mathrm{M}_{\mathrm{n}} \sim 90.000 \mathrm{~g} \mathrm{~mol}^{-1}$ ) that provide ductility and mechanical strength (Fig. 1a, d-e). Build-up of molecular weight during polymerisation is facilitated by additional reaction pathways of DEC-derived end groups, which

15 are not amenable with the common dimethyl carbonate reagent (Extended Data Fig. 2). PE-18,18 with desirable mechanical properties could be obtained by titanium-alkoxide polycondensation of stoichiometric amounts of the biorefinery-sourced $\mathrm{C}_{18}$ diester and diol (typically $\mathrm{M}_{\mathrm{w}} \sim 80.000 \mathrm{~g}$ $\mathrm{mol}^{-1}, \mathrm{M}_{\mathrm{n}} \sim 50.000 \mathrm{~g} \mathrm{~mol}^{-1}$ ). Both materials compare to commercial HDPE in their molecular weights (Fig. 1c), modulus, and ductility as determined by stress-strain experiments on specimens

20 generated by injection moulding, a widespread thermoplastic processing method for HDPE (Fig. 1d,e; further comparative literature values for HDPE are $\mathrm{E}_{\mathrm{y}}=900 \mathrm{MPa}, \sigma_{\mathrm{y}}=27 \mathrm{MPa}$ and for LDPE $\left.\mathrm{E}_{\mathrm{y}}=240 \mathrm{MPa}, \sigma_{\mathrm{y}}=12 \mathrm{MPa}\right){ }^{21}$ 

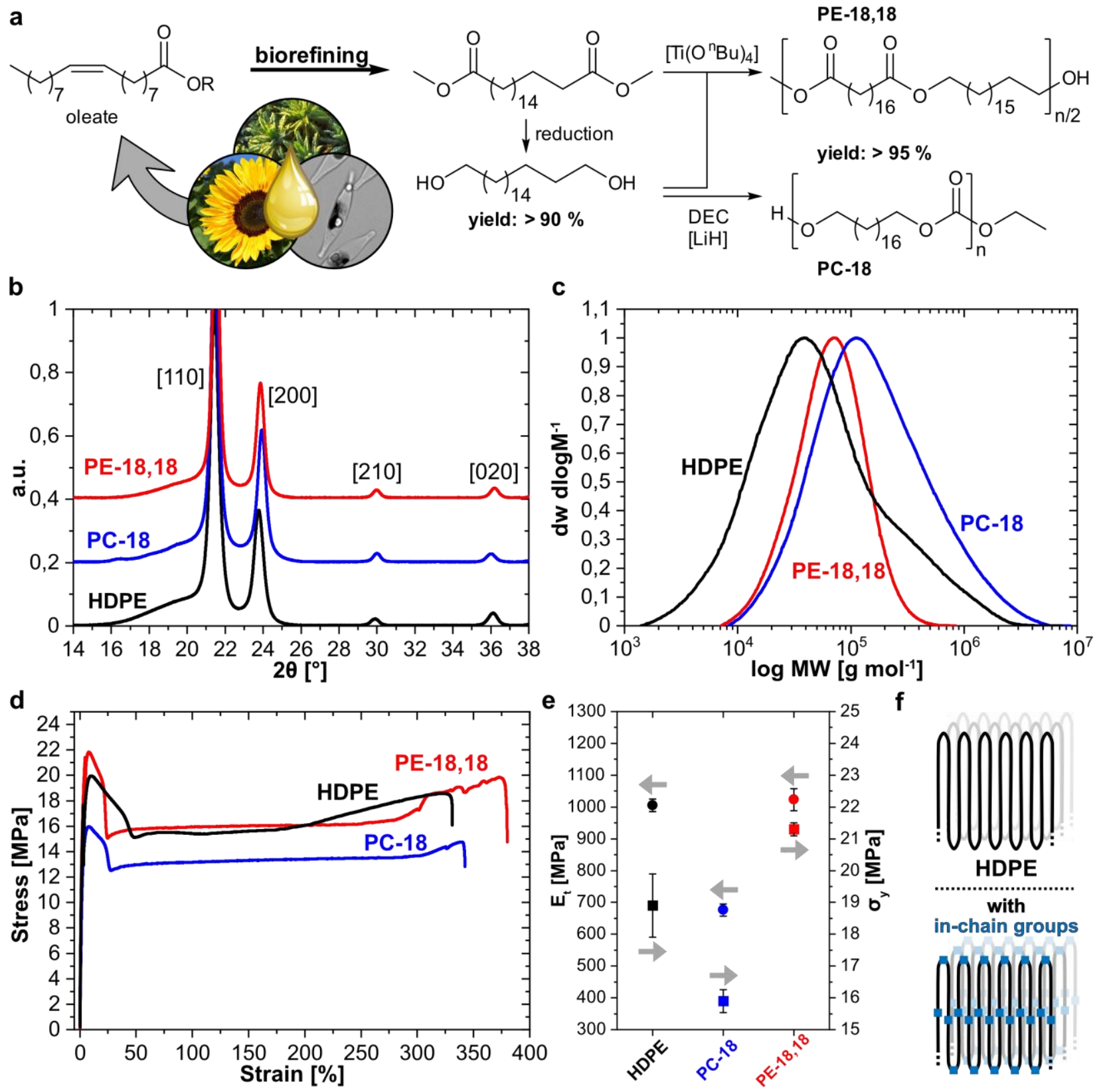

Fig. 1 | Preparation and characterisation of polyethylene-like polymers. a, Monomer synthesis starting from biorefining of plant or microalgae oils (examples shown: sunflowers, oil palms, microalgae Phaeodactylum tricornutum). b, WAXS of PC-18, PE-18,18 and commercial HDPE. Reflexes correspond to the orthorhombic unit cell of polyethylene in all cases. c, GPC traces of PC-18 and PE-18,18 in comparison to commercial HDPE. d, Representative stress-strain curves of injection moulded PC-18, PE-18,18 and commercial HDPE. e, Young's moduli and stress at yield values for PC-18, PE-18,18 and HDPE. Error bars shown are standard deviations. f, Schematic representation of the solid state structure of HDPE (top) and polyethylene-like polymers with in-chain functional groups (bottom). 
Wide angle X-ray scattering (WAXS) diffractograms of the materials prepared are virtually identical to that of HDPE (Fig. 1b), underlining a solid-state structure completely dominated by crystallisation of aligned hydrocarbon segments (Fig. 1f). Degrees of crystallinity of the polymers prepared as determined by deconvolution of WAXS data ( $c f$. SI for details) are essentially identical to the crystallinity of HDPE, with $\chi \approx 80 \%$. As expected, melting and crystallisation points of our PC-18 and PE-18,18 are lowered compared to HDPE due to the energy penalty ${ }^{15,22}$ resulting from incorporation of the in-chain functional groups in crystalline lamellae (Extended Data Fig. 3). Notwithstanding, crystallisation temperatures are sufficiently high for a facile melt processing, which is crucial for applications of plastics.

\section{Processing and fused filament fabrication}

As outlined, sample test bars were generated by standard melt processing methods, employing a twin-screw micro compounder and piston injection moulding. As a further, demanding processing method, additive manufacturing by fused filament fabrication was explored. For 3D-printing technologies, a closed-loop recycling with full retention of the materials' high-performance properties is particularly desirable.

For both our PC-18 and PE-18,18 materials, melt extrusion yielded filaments with an excellent dimensional accuracy within $\pm 0.02 \mathrm{~mm}$, matching the quality of commercial filaments (typically $\leq 0.05 \mathrm{~mm}$. Fig. 2a-c). Filaments of uncompromised quality could also be generated for compounds with colourants or carbon-fibres as an example of performance-enhancing fillers. 
a
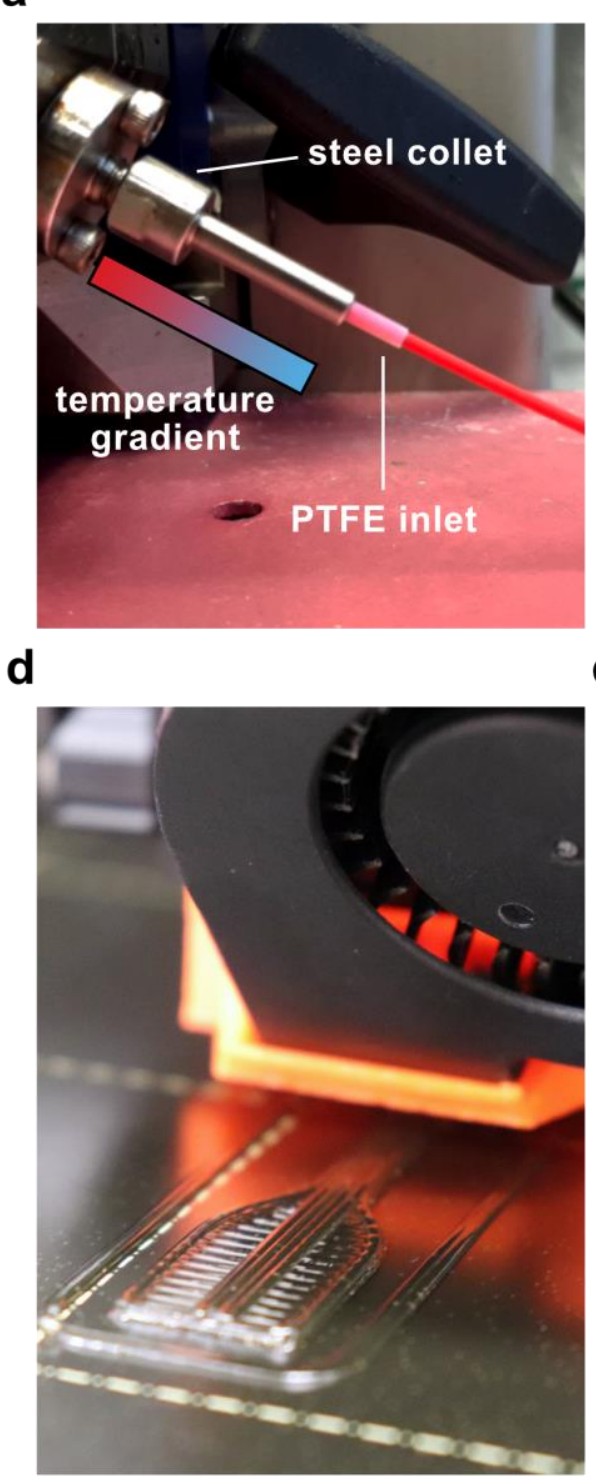

b

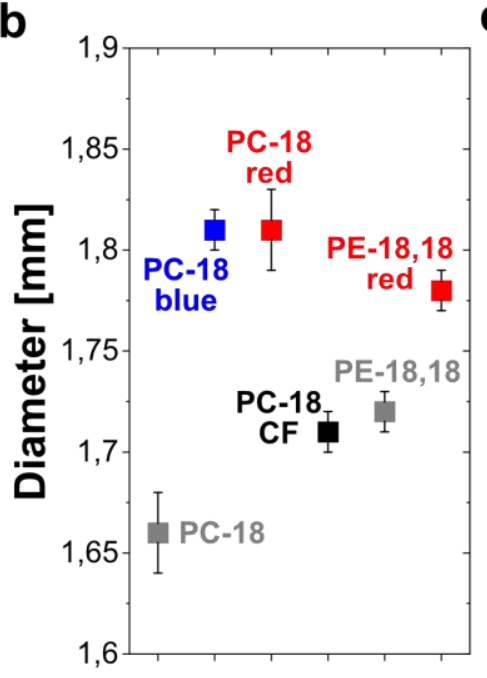

C

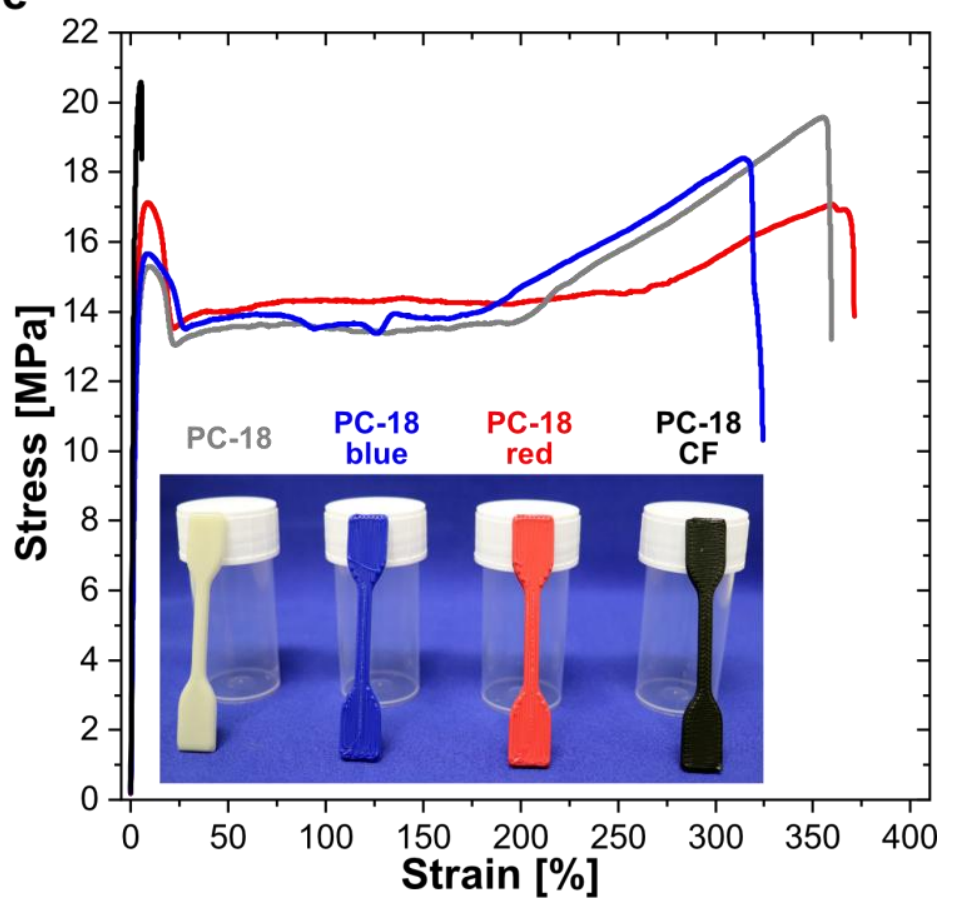

Fig. 2 Filament fabrication and 3D-printing of PC-18 and PE-18,18. a, Filament extrusion from a microcompounder, extrusion of red-coloured PE-18,18 shown. b, Diameter and dimensional accuracy of extruded filaments of all PC-18 and PE-18,18 compounds. Error bars shown are standard deviations. c, Spooled red and natural colour

filaments of PE-18,18. d, Printing of tensile test specimen. e, Stress-strain curves of 3D-printed tensile testing bars of PC-18 compounds. For clarity, a single exemplary measurement is shown for a given type of compound, see Extented Data Fig. 4 and the Supplementary Information for full datasets. Insert shows exemplary tensile testing specimens of each type of filament. White specimen/grey curve: natural PC-18. Red and blue specimen and curve: masterbatch coloured PC-18. Black: carbon-fibre reinforced PC-18. 
PC-18 and PE-18,18 were found to be well printable on typical standard printing surfaces like polyetherimide or glass (unlike HDPE which requires special surfaces ${ }^{23}$ ). This can be related to an improved compatibility with the polymer melt imparted by the polar in-chain groups. Note that in the solid-state, surface tension and wetting angles of these polymers do not differ significantly from 5 polyethylene ( $c f$. Supplementary Information), ester and carbonate groups being amenable to incorporation in polymer lamellae. This allowed for straightforward printing of tensile testing specimens using a standard commercial 3D-printer (Fig. 2d). Also a carbon-fibre reinforced $10 \mathrm{wt} \%$ blend of PC-18 was printable, without the necessity of harsher conditions. The 3D-printed testing bars exhibited excellent mechanical properties, namely a high ductility of up to $\varepsilon_{\mathrm{b}}=350 \%$ and Young's moduli of $\sim 640 \mathrm{MPa}$ for PC-18 (Fig. 2e). Equally, PE-18,18 specimens printed at the same conditions rival injection molded specimens with Young's moduli of $\sim 910 \mathrm{MPa}$ and $\varepsilon_{\mathrm{b}}$ $\sim 470 \%$ (Extended Data Fig. 4). In addition to the aforementioned tensile testing bars, more demanding 3D-prints could be realised as illustrated by a snap-on smartphone protective cover of red-coloured PE-18,18 (Fig. 3a). This everyday item is an example of a mechanically demanding

15 multiple use plastic object which calls for recyclability, in this case due to the limited life-time of a given smartphone generation. In terms of stability at elevated temperatures, a typical benchmark is stability at the boiling point of water. While the softening points of PC-18 and PE-18,18 are too low for such applications, it is worth noting that our polyethylene-like materials do provide a perspective also towards this target. From 1,48-tetraoctacontane diol, generated by catalytic chaindoubling of erucic acid, ${ }^{24}$ the diethyl carbonate polymerisation method yielded a high molecular weight $\left(\mathrm{M}_{\mathrm{w}} \sim 90.000 \mathrm{~g} \mathrm{~mol}^{-1}, \mathrm{M}_{\mathrm{n}} \sim 40.000 \mathrm{~g} \mathrm{~mol}^{-1}\right)$ ductile PC-48 with a narrow melt transition at $\mathrm{T}_{\mathrm{m}}=113{ }^{\circ} \mathrm{C}$ (Extended Data Fig. 3). A thin-walled $(0.4 \mathrm{~mm})$ cup 3D-printed from coloured PC-48 maintained its dimensions and shape upon repeated filling with boiling water (Fig. 3b). 
a

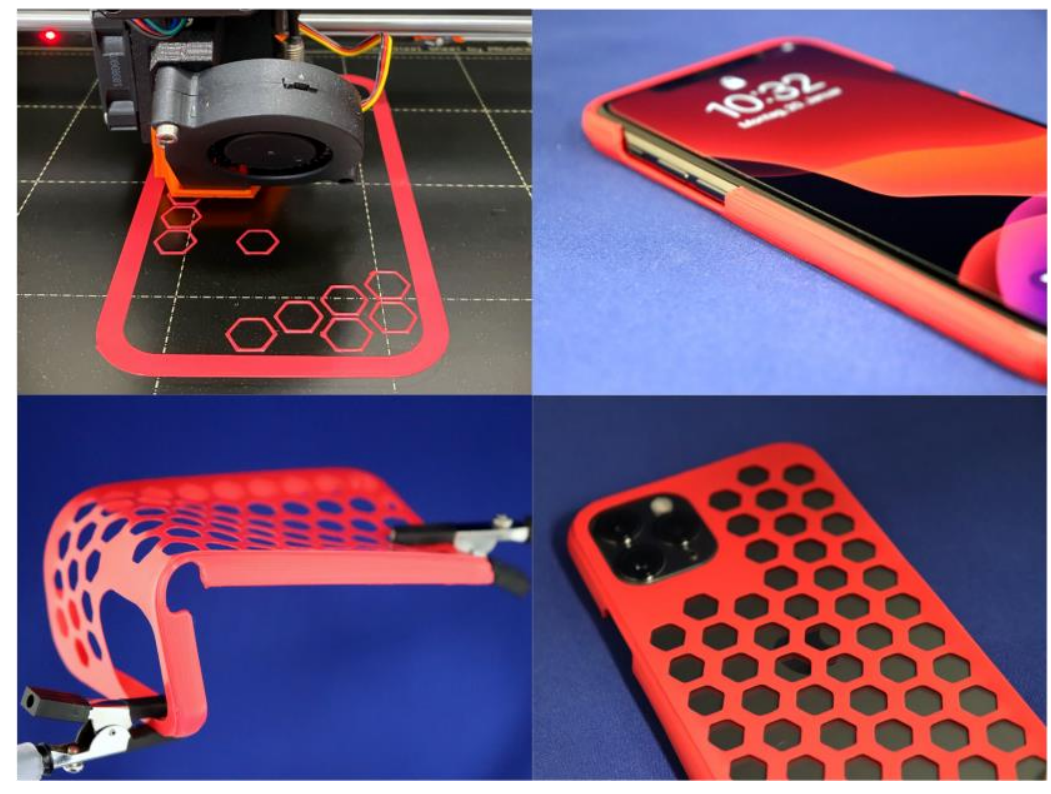

b

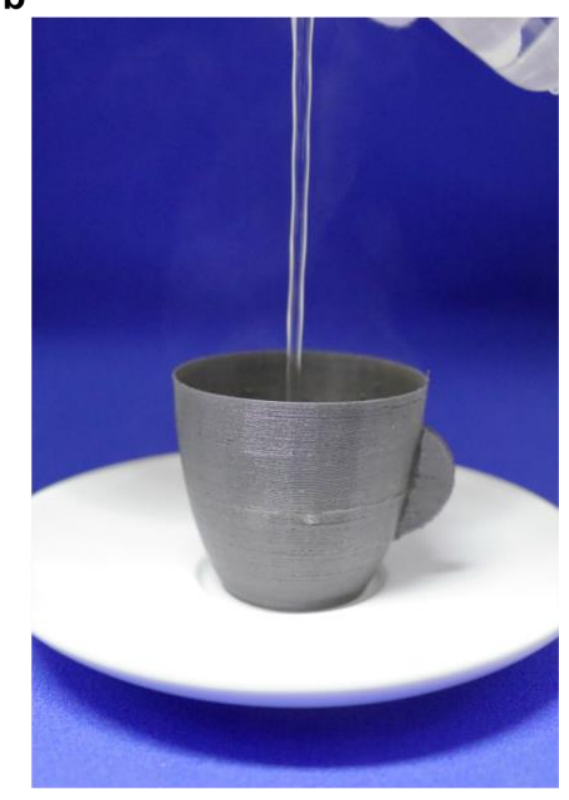

Fig. 3 | Exemplary 3D-prints. a, Snap-on smartphone protective cover printed with red-coloured PE-18,18. b, Boiling water $\left(>95^{\circ} \mathrm{C}\right)$ being poured into a 3D-printed cup of grey-coloured PC-48 as a probe of dimensional stability at elevated temperature

\section{Chemical recycling of polyethylene-like materials}

Both, long-chain polycarbonate and polyester, were amenable to quantitative chemical recycling under mild conditions in benign solvents (Fig. 4a). ${ }^{25}$ In case of PC-18, depolymerisation in basic EtOH solution yields the long-chain 1,18-octadecane diol. As a by-product of the chain breakdown,

10 diethyl carbonate is formed in stoichiometric amounts ( $c f$. Supplementary Information). Notably, the carbonate moiety accounts for only $5 \%$ of the carbon content in PC-18 (and 2\% in PC-48), yet the diethyl carbonate offers itself for re-use. Due to its volatility it can be readily separated and recovered from the reaction mixture by distillation. 

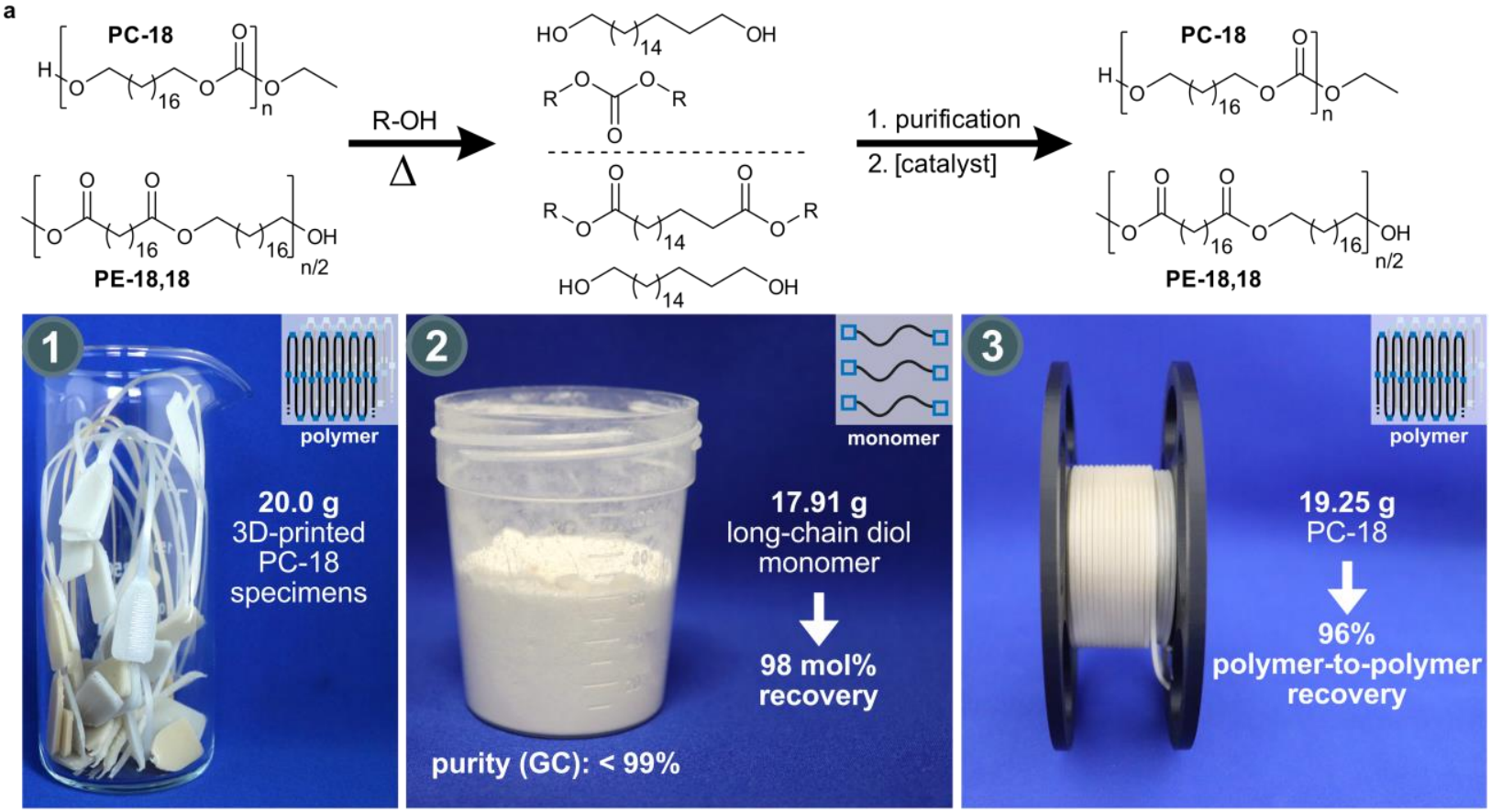

b
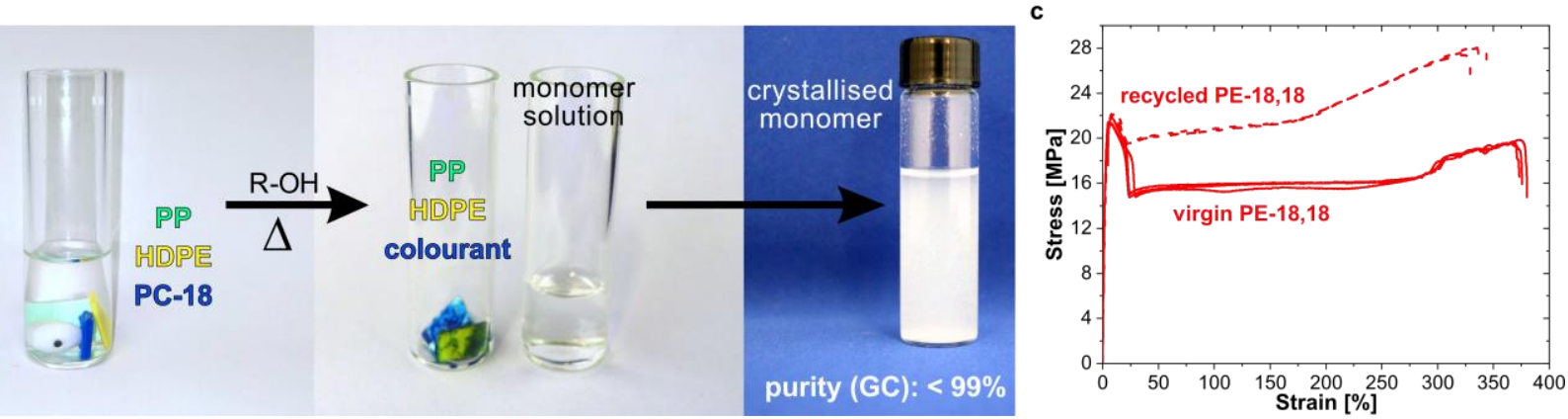

Fig. 4 | Chemical recycling of polyethylene-like materials. a, Schematic overview of the chemical recycling of PC18 and PE-18,18 via solvolysis and repolymerisation. Recycling experiment for PC-18; (1) $20.0 \mathrm{~g}$ of melt processed PC-18 employed for depolymerisation. (2) Depolymerisation at $120^{\circ} \mathrm{C}$ in EtOH (at $80 \mathrm{~g}$ polymer/L, $10 \mathrm{wt} \% \mathrm{KOH}$, $24 \mathrm{~h}$ ) yields high-quality monomer 1,18-octadecanediol in virtually quantitative yield. (3) PC-18 repolymerised from recovered monomer in $96 \%$ yield; the yield refers to the non-processed polymer due to inherent losses during melt processing; dimensions and dimensional accuracy of the recycled filament is identical to the initial polymer $(1.66 \mathrm{~mm}$ $\pm 0.02 \mathrm{~mm})$. b, Mixture of PP, HDPE and blue-coloured PC-18 as an example for a potential polyolefin waste stream, in $\mathrm{MeOH}$, and separated mixture after depolymerisation. The warm methanolic solution of 1,18-octadecane diol was separated and allowed to crystallise at room temperature for purification; purity was determined by GC. c, stress-strain curves of a virgin PE-18,18 batch and its recycled pendant. Note that the higher tensile strength as well as the strain hardening behavior of the recycled PE-18,18 in comparison to the virgin material can be related to the samples' higher molecular weight. 
A closed recycling loop was demonstrated by polymer-to-polymer recycling of $20.0 \mathrm{~g}$ of used PC-18 tensile testing bars in $250 \mathrm{~mL}$ of basic $\mathrm{EtOH}(10 \mathrm{wt} \% \mathrm{KOH})$. Employing a depolymerisation temperature of $120{ }^{\circ} \mathrm{C}$, complete depolymerisation occurred without the necessity for further measures, such as intense stirring or prior milling. A reaction time of $24 \mathrm{~h}$ was usually employed

5 to ensure complete conversion, but shorter reaction times of $1 \mathrm{~h}$ and possibly even less were also found to be sufficient to achieve a conversion $>99 \%$ (for details and other depolymerisation conditions $c f$. Extended Data Fig. 5). Note that complete depolymerisation could also be achieved without the need of a catalyst at $150{ }^{\circ} \mathrm{C}$ in $\mathrm{MeOH}$ within $24 \mathrm{~h}$. Upon cooling of the reaction mixture, the long-chain diol crystallised and was separated by filtration. A single recrystallisation afforded highly pure 1,18-octadecane diol (> 99\%) in virtually quantitative yield (17.91 g, corresponding to $98 \%$ ). By reacting the so-recovered monomer with diethyl carbonate again, $19.25 \mathrm{~g}$ of high molecular weight $\left(\mathrm{M}_{\mathrm{w}} \sim 240.000 \mathrm{~g} \mathrm{~mol}^{-1}, \mathrm{M}_{\mathrm{n}} \sim 70.000 \mathrm{~g} \mathrm{~mol}^{-1}\right)$ PC-18 could be obtained, which translates to a chemical polymer-to-polymer recycling rate of $96 \%$ (Fig. 4a). As anticipated, this recycled PC-18 retained its materials and processing quality (Extended Data Fig. 6).

15 In a real-life sorting of mixed plastic waste streams, separation occurs physically by sink-flow density separation or mechanical sorting after NIR analysis. ${ }^{10}$ Both methods could include our polyethylene-like polymers in the polyolefin stream together with polypropylene (PP) and polyethylene. Thus, the ability to separate our polyethylene-like polymers from these polyolefins in the chemical recycling process is desirable. To this end, pieces of commercial PP (re-usable hot 20 beverage cup), HDPE (solvent bottle cap) and of blue-coloured PC-18 were subjected to the above depolymerisation process (Fig. 4b and Extended Data Fig. 7). While the polyethylene-like PC-18 was completely depolymerised and dissolved, the PP and HDPE pieces were recovered in their original unaltered state. From work-up of the filtrate by crystallisation, neat 1,18-octadecane diol (GC: > 99\%) was recovered quantitatively (98\% yield) again. Given that poly(ethylene 
terephthalate) (PET) is also a major plastic present in waste streams and subject to recycling, the ability to recycle our polymers from PET is desirable e.g. to avoid contaminations of either stream. Notably, PC-18 can be depolymerised completely to monomer while PET $\left(\mathrm{T}_{\mathrm{m}}=268^{\circ} \mathrm{C}\right)$ is retained and the materials can be separated quantitatively (Extended Data Fig. 7).

5 In contrast to the long-chain polycarbonates, where depolymerisation yields the diol as the only long-chain compound, depolymerisation of PE-18,18 yields a mixture of two non-volatile longchain monomers, diester and diol. This mixture cannot be separated easily, but it offers itself for a direct repolymerisation to new PE-18,18. Depolymerisation of PE-18,18 in $\mathrm{MeOH}$ proceeded without the necessity of a catalyst, and complete depolymerisation was achieved within $12 \mathrm{~h}$ (or

$102 \mathrm{~h}$ with catalyst) at $150{ }^{\circ} \mathrm{C}$ at a concentration of $40 \mathrm{~g}$ polymer/L. Furthermore, the solvolysis process even allowed for the selective depolymerisation and recovery of the PE-18,18 component from a melt blend with HDPE, leaving the HDPE matrix intact and thereby facilitating its isolation (Extended Figure 8).

Note that although the polymers can be depolymerised as outlined, at ambient conditions typical

15 for applications, the polymers are not unfavourably sensitive and no evidence of decomposition is observed over months ( $c f$. Supplementary Information).

Removal of $\mathrm{MeOH}$ in vacuo from the depolymerisation solution yielded a mixture of $\mathrm{C}_{18}$ diester and $\mathrm{C}_{18}$ diol in a precise 1:1 ratio (as evidenced by ${ }^{1} \mathrm{H}-\mathrm{NMR}$ spectroscopy; Supplementary Information). Without further workup, this mixture was repolymerised with $\left[\mathrm{Ti}\left(\mathrm{O}^{\mathrm{n}} \mathrm{Bu}\right)_{4}\right]$ catalyst to yield high molecular weight PE-18,18 (Extended Data Fig. 9). The thermal and particularly the materials properties of the so-recycled polymer were on par with virgin PE-18,18, as evidenced by tensile testing of both injection moulded specimens as well as specimens generated by 3D-printing (cf. Fig. 4c). 
Notably, depolymerisation for chemical recycling is also readily possible by hydrolysis in water as a reaction medium, despite the heterogeneous nature of the aqueous reaction mixtures with the hydrophobic polymer and monomer. Particularly, polycarbonate PC-18 was completely converted to clean 1,18 -diol at $180{ }^{\circ} \mathrm{C}$ under basic conditions $(\mathrm{KOH})$, the carbonate moieties being released 5 as carbon dioxide (Extended Data Fig. 5).

In summary, we have fully demonstrated a closed-loop recycling approach for polyethylene-like materials. Key are low densities of break points in the polyethylene chain such that the crystalline structure is not affected, and consequently properties like mechanical strength are not compromised. Chemical recycling of polyethylene by breaking of $\mathrm{C}-\mathrm{C}$ bonds requires $\Delta \mathrm{H} \approx 3 \mathrm{MJ}$

$10 \mathrm{~kg}^{-1}$ reaction energy alone and heating to $800{ }^{\circ} \mathrm{C}$, with ethylene being obtained with a limited selectivity. By contrast, the alcoholysis reactions used here are essentially thermoneutral equilibrium reactions shifted to depolymerisation by an excess of solvent. Due to the low density of break points, the amount of reagent, here an alcohol, that is cycled in this depolymerisation/polymerisation is low (e.g. 11 wt.-\% in methanolysis of PE-18,18).

15 Appropriate straightforward polymerisation protocols yield long-chain polycarbonates or polyesters with high molecular weights reflecting in desirable materials properties, namely ductility and rigidity. Depolymerisation of long-chain polycarbonates and alternatively of longchain polyesters occurs under mild conditions $\left(120{ }^{\circ} \mathrm{C}\right.$ in $\mathrm{EtOH}$ or $\left.\mathrm{MeOH}\right)$ and yields highly pure monomers from recrystallisation, as demonstrated in detail for PC-18 and PE-18,18. As break

20 points in the chain, carbonate groups are particularly attractive as they yield crystallisable, longchain diols and volatile dialkyl carbonates that can be easily separated. Repolymerisation yields materials with uncompromised quality with a polymer-to-polymer recycling efficiency of $96 \%$. Such a quantitative recovery was also possible selectively from mixtures with commercial 
polypropylene and polyethylene (HDPE), which would occur in waste plastic streams and also seperation from PET was possible. Colourants and also carbon-fibres, which even in small amounts are detrimental to recycling in general, could be removed completely. In addition to injection moulding, as a common wide-spread processing method that serves numerous applications, the

5 polyethylene-like polymers are also well-suited for 3D-printing as a complementary manufacturing method that would also benefit particularly from chemical recycling.

Plant oils are employed as the original feedstock because their long methylene sequences are a convenient source of the long-chain monomers used. Notably, in addition to the commercial biorefining of palm oil by olefin metathesis, enzymatic $\omega$-oxidation ${ }^{26}$ or carbonylation of

10 traditional plant oils or microalgae oils ${ }^{27}$ offer themselves as an alternative technology base to access the virgin polymer materials. At present, the long-chain monomers are not available at a similar price and scale as ethylene, which is the cheapest building block of the chemical industry.

The approach demonstrated provides a perspective for fully recyclable polyethylene-like materials suitable for high quality applications. This is an important potential building block for a resource-

15 saving low emission economy beyond the currently used technology.

\section{ONLINE CONTENT}

Any methods, additional references, Nature Research reporting summaries, source data, extended data, supplementary information, acknowledgements, peer review information; details of author contributions and competing interests; and statements of data and code availability are available at https://doi.org/10.1038/s41586-020-03149-9 
1. Vollmer, I. et al. Beyond Mechanical Recycling: Giving New Life to Plastic Waste. Angew. Chem. Int. Ed.; 10.1002/anie.201915651 (2020).

2. Ellen MacArthur Foundation. The New Plastics Economy. Available at https://www.ellenmacarthurfoundation.org/assets/downloads/EllenMacArthurFoundation_TheNewPla sticsEconomy_Pages.pdf (2016).

3. Hees, T., Zhong, F., Stürzel, M. \& Mülhaupt, R. Tailoring Hydrocarbon Polymers and AllHydrocarbon Composites for Circular Economy. Macromol. Rapid Commun. 40, e1800608; 10.1002/marc.201800608 (2019).

4. Hoe, G. X. de et al. Sustainable Polyester Elastomers from Lactones: Synthesis, Properties, and Enzymatic Hydrolyzability. J. Am. Chem. Soc. 140, 963-973; 10.1021/jacs.7b10173 (2018).

5. Rahimi, A. \& García, J. M. Chemical recycling of waste plastics for new materials production. Nat. Rev. Chem. 1, 46; 10.1038/s41570-017-0046 (2017).

6. Zhang, X., Fevre, M., Jones, G. O. \& Waymouth, R. M. Catalysis as an Enabling Science for Sustainable Polymers. Chem. Rev. 118, 839-885; 10.1021/acs.chemrev.7b00329 (2018).

15 7. Zhu, Y., Romain, C. \& Williams, C. K. Sustainable polymers from renewable resources. Nature 540, 354-362; 10.1038/nature21001 (2016).

8. Coates, G. W. \& Getzler, Y. D. Y. L. Chemical recycling to monomer for an ideal, circular polymer economy. Nat. Rev. Mater.; 10.1038/s41578-020-0190-4 (2020).

9. Eagan, J. M. et al. Combining polyethylene and polypropylene: Enhanced performance with PE/iPP multiblock polymers. Science 355, 814-816; 10.1126/science.aah5744 (2017).

10. Ragaert, K., Delva, L. \& van Geem, K. Mechanical and chemical recycling of solid plastic waste. Waste Manag. 69, 24-58; 10.1016/j.wasman.2017.07.044 (2017). 
11. Lopez, G., Artetxe, M., Amutio, M., Bilbao, J. \& Olazar, M. Thermochemical routes for the valorization of waste polyolefinic plastics to produce fuels and chemicals. A review. Renew. Sustain. Energ. Rev. 73, 346-368; 10.1016/j.rser.2017.01.142 (2017).

12. Stürzel, M., Mihan, S. \& Mülhaupt, R. From Multisite Polymerization Catalysis to Sustainable Materials and All-Polyolefin Composites. Chem. Rev. 116, 1398-1433; 10.1021/acs.chemrev.5b00310 (2016).

13. Strobl, G. R. The Physics of Polymers (Springer, Berlin, 2007).

14. Kaminsky, W. Production of Polyolefins by Metallocene Catalysts and Their Recycling by Pyrolysis. Macromol. Symp. 360, 10-22; 10.1002/masy.201500127 (2016).

15. Stempfle, F., Ortmann, P. \& Mecking, S. Long-Chain Aliphatic Polymers To Bridge the Gap between Semicrystalline Polyolefins and Traditional Polycondensates. Chem. Rev. 116, 4597-4641; 10.1021/acs.chemrev.5b00705 (2016).

16. Chikkali, S. \& Mecking, S. Refining of plant oils to chemicals by olefin metathesis. Angew. Chem. Int. Ed. 51, 5802-5808; 10.1002/anie.201107645 (2012).

15 17. Press release by Elevance Renewable Sciences of July 18, 2013 (2013)

18. Biermann, U., Bornscheuer, U., Meier, M. A. R., Metzger, J. O. \& Schäfer, H. J. Oils and fats as renewable raw materials in chemistry. Angew. Chem. Int. Ed. 50, 3854-3871; 10.1002/anie.201002767 (2011).

19. Ortmann, P., Heckler, I. \& Mecking, S. Physical properties and hydrolytic degradability of polyethylene-like polyacetals and polycarbonates. Green Chem. 16, 1816-1827; 10.1039/C3GC42592D (2014).

20. Mutlu, H., Hofsäß, R., Montenegro, R. E. \& Meier, M. A. R. Self-metathesis of fatty acid methyl esters: full conversion by choosing the appropriate plant oil. $R S C A d v .3,4927 ; 10.1039 / \mathrm{c} 3 \mathrm{ra} 40330 \mathrm{k}$ (2013). 
21. Jeremic, D. in Ullmann's Encyclopedia of Industrial Chemistry, edited by Wiley-VCH Verlag GmbH \& Co. KGaA (2014), pp. 1-42.

22. Sanchez, I. C. \& Eby, R. K. Thermodynamics and Crystallization of Random Copolymers. Macromolecules 8, 638-641; 10.1021/ma60047a012 (1975).

23. Schirmeister, C. G., Hees, T., Licht, E. H. \& Mülhaupt, R. 3D printing of high density polyethylene by fused filament fabrication. Addit. Manuf. 28, 152-159; 10.1016/j.addma.2019.05.003 (2019).

24. Witt, T., Häußler, M., Kulpa, S. \& Mecking, S. Chain Multiplication of Fatty Acids to Precise Telechelic Polyethylene. Angew. Chem. Int. Ed. 56, 7589-7594; 10.1002/anie.201702796 (2017).

25. Capello, C., Fischer, U. \& Hungerbühler, K. What is a green solvent? A comprehensive framework for the environmental assessment of solvents. Green Chem. 9, 927; 10.1039/b617536h (2007).

26. Huf, S., Krügener, S., Hirth, T., Rupp, S. \& Zibek, S. Biotechnological synthesis of long-chain dicarboxylic acids as building blocks for polymers. Eur. J. Lipid Sci. Technol. 113, 548-561; 10.1002/ejlt.201000112 (2011).

27. Hess, S. K., Lepetit, B., Kroth, P. G. \& Mecking, S. Production of chemicals from microalgae lipids status and perspectives. Eur. J. Lipid Sci. Technol. 120, 1700152; 10.1002/ejlt.201700152 (2018). 


\section{METHODS}

\section{Materials}

All chemicals were used as received without further purification. 1,18-octadecanedioic acid was purchased from Elevance Renewable Sciences Inc. Titanium(IV) n-butoxide (97 \%), lithium

5 aluminium hydride (95\%), lithium hydride (95\%), diethyl carbonate $(\geq 99 \%)$, potassium hydroxide $(\geq 99 \%)$ and methanol $(\geq 99.8 \%)$ were purchased from Sigma Aldrich. Ethanol $(\geq$ $99.8 \%$ ), xylene (isomeric mixture, $\geq 99 \%$ ) and tetrahydrofuran $(\geq 99.5 \%$ ) were purchased from Carl Roth. 2-Propanol ( $\geq 99.7 \%$ ) was purchased from VWR and toluene ( $\geq 99.9 \%)$ was purchased from Merck. $\mathrm{N}, \mathrm{O}$-Bis(trimethylsilyl)trifluoroacetamide (BSTFA) (98\%) was purchased from abcr.

10 High-density polyethylene (HDPE) (analogous to industrial grade Lyondellbasell Alathon H5112) was purchased from Sigma Aldrich. Omnidynamics PLA/ABS colourant masterbatch in red and blue was used for the colouration of the 3D-printing filament. Carbon-fibres from $R \& G$ Faserverbundwerkstoffe $\mathrm{GmbH}$ were used for the fabrication of reinforced 3D-printing filament. Deuterated solvents for NMR spectroscopy were purchased from Eurisotop and dried over 15 molecular sieves from Riedel de-Haën $(0.4 \mathrm{~nm})$.

All manipulations involving air- and/or moisture-sensitive substances were carried out under inert atmosphere using standard Schlenk and glovebox techniques.

\section{Characterisation and processing techniques}

20 Nuclear magnetic resonance (NMR) spectra were recorded on a Bruker Avance III HD 400 spectrometer. Chemical shifts were referenced to the signal of the solvent (residual proton 
resonances for ${ }^{1} \mathrm{H}$ spectra, carbon resonances for ${ }^{13} \mathrm{C}$ spectra). Mestrenova software by Mestrelab Research S.L. (version 14.0.0) was used for data evaluation.

Infrared spectra (IR) of polymer samples were recorded on a Perkin Elmer Spectrum 100 FT-IR attenuated total reflection spectrometer. Spectrum software by Perkin Elmer (version 6.2.0) was 5 used for data evaluation.

Gas chromatography (GC) was carried out on a Perkin Elmer Clarus 500 instrument with an autosampler and flame ionisation detection (FID) on a Perkin Elmer Elite-5 capillary column (5\% diphenyl, $95 \%$ dimethylpolysiloxane, length: $30 \mathrm{~m}$, inner diameter: $0.25 \mathrm{~mm}$, film thickness: $0.25 \mu \mathrm{m}$ ), using helium as carrier gas at a flow rate of $1.5 \mathrm{~mL} \mathrm{~min}^{-1}$. A temperature programme of 10 an initial isothermal step of $1 \mathrm{~min}$ at $70{ }^{\circ} \mathrm{C}$, followed by heating with $15^{\circ} \mathrm{C} \min ^{-1}$ to $250{ }^{\circ} \mathrm{C}$, and 5 min isothermal at this temperature was used, with an injector temperature of $300{ }^{\circ} \mathrm{C}$ and a detector temperature of $320^{\circ} \mathrm{C}$. Diols were derivatised by silylation using BSTFA for GC analysis.

Molecular weights of the polymers were determined by high temperature gel permeation chromatography (GPC) in 1,2-dichlorobenzene at $160^{\circ} \mathrm{C}$ on a Polymer Char GPC-IR instrument,

15 equipped with PSS Polefin Linear XL columns ( $3 \times 30 \mathrm{~cm}$, additional guard column), an infrared detector (IR5 MCT, concentration signal) and a viscosity detector. A standard flow rate of $0.5 \mathrm{~mL}$ $\min ^{-1}$ was used. Molecular weights were determined via universal calibration versus narrow polystyrene standards from PSS Polymer Standards (software: PSS WinGPC, version 8.32). MarkHouwink parameters determined for polystyrene and used in the data analysis are $\alpha=0.703$ and $\mathrm{K}$ $20=0.01522 \mathrm{~mL} \mathrm{~g}^{-1}$. Mark-Houwink parameters of PC-18 and PE-18,18 were determined from a Mark-Houwink plot of intrinsic viscosity $v s$. elution volume. 
Differential scanning calorimetry (DSC) measurements of polymers were carried out on a Netzsch DSC 204 F1 instrument (software: Netzsch Proteus Thermal Analysis, version 6.1.0) with a heating/cooling rate of $10 \mathrm{~K} \mathrm{~min}^{-1}$. Data reported are from second heating cycles.

Scanning electron microscopy (SEM) images were recorded on a Zeiss Gemini 500 microscope by 5 secondary electron detection (SE2) with an acceleration voltage of $3 \mathrm{kV}$. Polymer samples were sputtered with a $6 \mathrm{~nm}$ platinum layer using a Quorum Q150 sputter coater.

Injection moulded test specimens were prepared using a Xplore IM 5.5 micro injection moulder. The cylinder and mould temperature were set to $180{ }^{\circ} \mathrm{C}$ and $60{ }^{\circ} \mathrm{C}$, respectively, and an injection pressure of 16 bar for $10 \mathrm{~s}$ and 12 bar for $15 \mathrm{~s}$ was applied. Prior to injection moulding, the polymers

10 were homogenised under vacuum in a Xplore MC 5 micro compounder at $180{ }^{\circ} \mathrm{C}$ and $50 \mathrm{rpm}$ for $30 \mathrm{~min}$.

3D printing (fused filament fabrication) of tensile test specimens and demonstration objects was conducted using a Prusa i3 MK3 printer.

Tensile tests were performed on a Zwick Z005/1446 Retroline tC II instrument at a crosshead speed of $5 \mathrm{~mm} \mathrm{~min}^{-1}$ on 3D-printed or injection moulded tensile testing specimens (ISO 527-2, type 5A). The determination of the Young's modulus was conducted at a crosshead speed of $1 \mathrm{~mm} \mathrm{~min}^{-1}$. Prior to tensile testing, the samples were preconditioned at room temperature. The Zwick Roell testXpert software version 11.0 was used for data evaluation.

Wide angle x-ray scattering (WAXS) diffractograms were recorded on a D8 Discover instrument 20 (Bruker) with a Vantec (Bruker) detector. Polymer crystallinities ( $\chi$ WAXs) were determined from the WAXS patterns as: $\chi_{\mathrm{WAXS}}=\left[\mathrm{A}_{\mathrm{c}}(110)+\mathrm{A}_{\mathrm{c}}(200)\right] /\left[\mathrm{A}_{\mathrm{c}}(110)+\mathrm{A}_{\mathrm{c}}(200)+\mathrm{A}_{\mathrm{a}}\right]$ where $\mathrm{A}_{\mathrm{c}}$ refers to the 
integrated area of the Bragg reflections from the orthorhombic PE crystal and $\mathrm{A}_{\mathrm{a}}$ to the integrated area of the amorphous halo. A Voigt fit was used.

The surface interfacial tensions of PC-18, PE-18,18 and commercial HDPE were determined by the method of Fowkes ${ }^{28}$ on a drop shape analyzer DSA25 by KRÜSS.

5 Error bars shown are standard deviations from three to eight independent measurements (tensile properties) or twenty independent measurements (filament diameters), respectively.

\section{Polymerisation experiments}

In a typical polycondensation for PC-18, 1,18-octadecane diol (1.0 equiv.) together with a stirring

10 bar was dried in a three-necked Schlenk tube at $60{ }^{\circ} \mathrm{C}$ in vacuo. A cooled condensor flask that allows for monitoring of the volatiles released from the polymerisation mixture was connected, diethyl carbonate (2.5 equiv.) was added in a nitrogen stream and the temperature was raised to $120{ }^{\circ} \mathrm{C}$ (stirring at $\left.500 \mathrm{rpm}\right)$. When a homogenous mixture was obtained, $\mathrm{LiH}(0.5 \mathrm{~mol} \%)$ was added as a suspension in diethyl carbonate. Typically, $15-45$ min after the addition of $\mathrm{LiH}$

15 condensation of ethanol was observed. When the condensation of ethanol had ceased, vacuum was applied by means of a membrane pump connected to the condensor to continue the oligomerisation in vacuo. The pressure was gradually decreased from 900 mbar to 10 mbar over the course of $1 \mathrm{~h}$ while the temperature was increased to $180{ }^{\circ} \mathrm{C}$. In the following polymerisation step, high vacuum (ca. $10^{-2}$ mbar) was applied via the Schlenk line, connected to a rotary vane oil pump. The 20 polycondensation was continued for typically $16 \mathrm{~h}$. To facilitate recovery from the vessel, the polymer melt was diluted with xylene at $120{ }^{\circ} \mathrm{C}$ and the polymer was precipitated by pouring the 
solution into $-30{ }^{\circ} \mathrm{C}$ 2-propanol, washed with 2-propanol and dried in a vacuum oven at $50{ }^{\circ} \mathrm{C}$ to afford PC-18.

The polycondensation to PC-48 was conducted analogous to the procedure for PC-18. 1,48octadecane diol (1.0 equiv.), synthesised from erucic acid by a reported catalytic procedure ${ }^{24}$ was

5 dried in a three-necked Schlenk tube at $100{ }^{\circ} \mathrm{C}$ under vacuum. Diethyl carbonate (5.0 equiv.) and $\mathrm{LiH}(1.0 \mathrm{~mol} \%)$ were added. After the oligomerisation step, the polymerisation step was conducted for $72 \mathrm{~h}$ at $180^{\circ} \mathrm{C}$ under high vacuum. The polymer was diluted with xylene at $160{ }^{\circ} \mathrm{C}$, and worked up as described for PC-18.

For the polyesterification ${ }^{29,30}$ to PE-18,18, the same instrumentation was applied as for

10 polycarbonate-18. 1,18-octadecane diol (1.0 equiv.) and 1,18-dimethyl octadecanedioate (1.0 equiv.) were dried in a three-necked Schlenk tube at $60{ }^{\circ} \mathrm{C}$ under vacuum. A toluene solution $(0.028 \mathrm{M})$ of titanium(IV) n-butoxide (0.084 mol\% vs. monomer) was added and the temperature was raised to $180^{\circ} \mathrm{C}$ (stirring at $350 \mathrm{rpm}$ ). Oligomerisation commenced, and vacuum was gradually applied (900 mbar to 10 mbar) over the course of $6 \mathrm{~h}$. The polymerisation step was conducted at

$15180{ }^{\circ} \mathrm{C}$ for typically $18 \mathrm{~h}$. The resulting polymer was dissolved at $160{ }^{\circ} \mathrm{C}$ in xylene, precipitated in $-30{ }^{\circ} \mathrm{C}$ 2-propanol and worked up as described for PC-18.

\section{Larger-Scale Depolymerisation Experiments}

In a $500 \mathrm{~mL}$ steel pressure vessel, $20.0 \mathrm{~g}$ of melt processed PC-18 were subjected to ethanolysis 20 (250 mL ethanol, $2.0 \mathrm{~g} \mathrm{KOH})$ by stirring $(500 \mathrm{rpm})$ for $24 \mathrm{~h}$ at $120^{\circ} \mathrm{C}$. The methanolysis of $25.0 \mathrm{~g}$ melt processed PE-18,18 (400 mL methanol) was conducted accordingly by stirring for $24 \mathrm{~h}$ at $120^{\circ} \mathrm{C}$. 
Concentration in vacuo of the depolymerisation solution of PC-18, crystallisation at $-30{ }^{\circ} \mathrm{C}$ and finally recrystallisation from methanol at $-30{ }^{\circ} \mathrm{C}$ yielded 1,18 -octadecane diol in high purity (17.9 g, $98 \mathrm{~mol} \%, \mathrm{GC}>99 \%)$.

Complete removal of methanol from the depolymerisation solution of PE-18.18 under reduced pressure yielded a repolymerisable, stoichiometric 1:1 mixture of the two monomers (27.6 g, $99 \mathrm{~mol} \%$ ). In case of the depolymerisation of coloured PE-18,18, the colourant solidified upon cooling of the depolymerisation mixture and was removed from the monomer solution.

Repolymerisation of the so recovered monomers was conducted as described in the section 'Polymerisation experiments'.

\section{Separation from waste streams}

To simulate seperation from a potential waste stream, methanolysis ( $5 \mathrm{~mL}$ methanol, $20 \mathrm{mg} \mathrm{KOH}$ ) of coloured PC-18 (200 mg) was conducted in the presence of samples of HDPE and polypropylene (PP) at $100{ }^{\circ} \mathrm{C}$ for $24 \mathrm{~h}$. The polyolefin samples remained unaltered during the solvolysis process (cf. Extended Data Fig. 7) and were seperated by siphoning off the monomer solution. 1,1815 octadecane diol was recovered by crystallisation in high purity (180 mg, $98 \mathrm{~mol} \%$, GC > $99 \%)$.

Likewise, PP samples added to the above larger-scale depolymerisation of PE-18,18 at $120{ }^{\circ} \mathrm{C}$ in methanol for $24 \mathrm{~h}$ remained unaltered.

Analogous to the separation from polyolefins, coloured PC-18 was depolymerised in the presence of polyethylene terephthalate in methanol at $150{ }^{\circ} \mathrm{C}$ for $24 \mathrm{~h}$. The solid polyethylene terephthalate sample remained intact (cf. Extended Data Figure 7) and was removed from the monomer solution. Neat 1,18-octadecane diol was recovered by crystallisation. 


\section{DATA AVAILABILITY}

The data that support the findings of this study are available from the corresponding author on request.

28. Fowkes, F. M. Attractive Forces at Interfaces. Industrial \& Engineering Chemistry 56, 40-52; 10.1021/ie50660a008 (1964).

29. Stempfle, F., Quinzler, D., Heckler, I. \& Mecking, S. Long-Chain Linear C 19 and C 23 Monomers and Polycondensates from Unsaturated Fatty Acid Esters. Macromolecules 44, 4159-4166; 10.1021/ma200627e (2011).

30. Otera, J. \& Nishikido, J. Esterification. Methods, reactions, and applications. 2nd ed. (Wiley-VCH, Weinheim, 2010).

31. Sun, J., Aly, K. I. \& Kuckling, D. A novel one-pot process for the preparation of linear and hyperbranched polycarbonates of various diols and triols using dimethyl carbonate. $R S C A d v .7$, 12550-12560; 10.1039/C7RA01317E (2017).

32. AlAbbad, M., Giri, B. R., Szori, M., Viskolcz, B. \& Farooq, A. A high temperature kinetic study for the thermal unimolecular decomposition of diethyl carbonate. Chem. Phys. Lett. 684, 390-396; 10.1016/j.cplett.2017.07.020 (2017).

\section{ACKNOWLEDGMENTS}

We thank Manuel Schnitte and Lars Bolk for GPC and DSC measurements. Support of our studies on

20 degradable polyethylenes by the ERC (Advanced Grant DEEPCAT, No. 832480) is gratefully acknowledged. 


\section{AUTHOR CONTRIBUTIONS}

S.M. conceived the concept. M.E., M.H. and S.M. jointly devised the experimental programm. M.H. and D.R. performed experiments with polycarbonates and 3D-printing. M.E. performed experiments with polyesters. M.H., M.E., and S.M. wrote the manuscript. M.H. and M.E. contributed equally to this work.

\section{COMPETING FINANCIAL INTERESTS}

A patent has been filed by the University of Konstanz on findings reported here.

\section{ADDITIONAL INFORMATION}

Supplementary Information is available for this paper at https://doi.org/ https://doi.org/10.1038/s41586020-03149-9

10 Correspondence and requests for materials should be addressed to S.M. 


\section{EXTENDED DATA FIGURES}

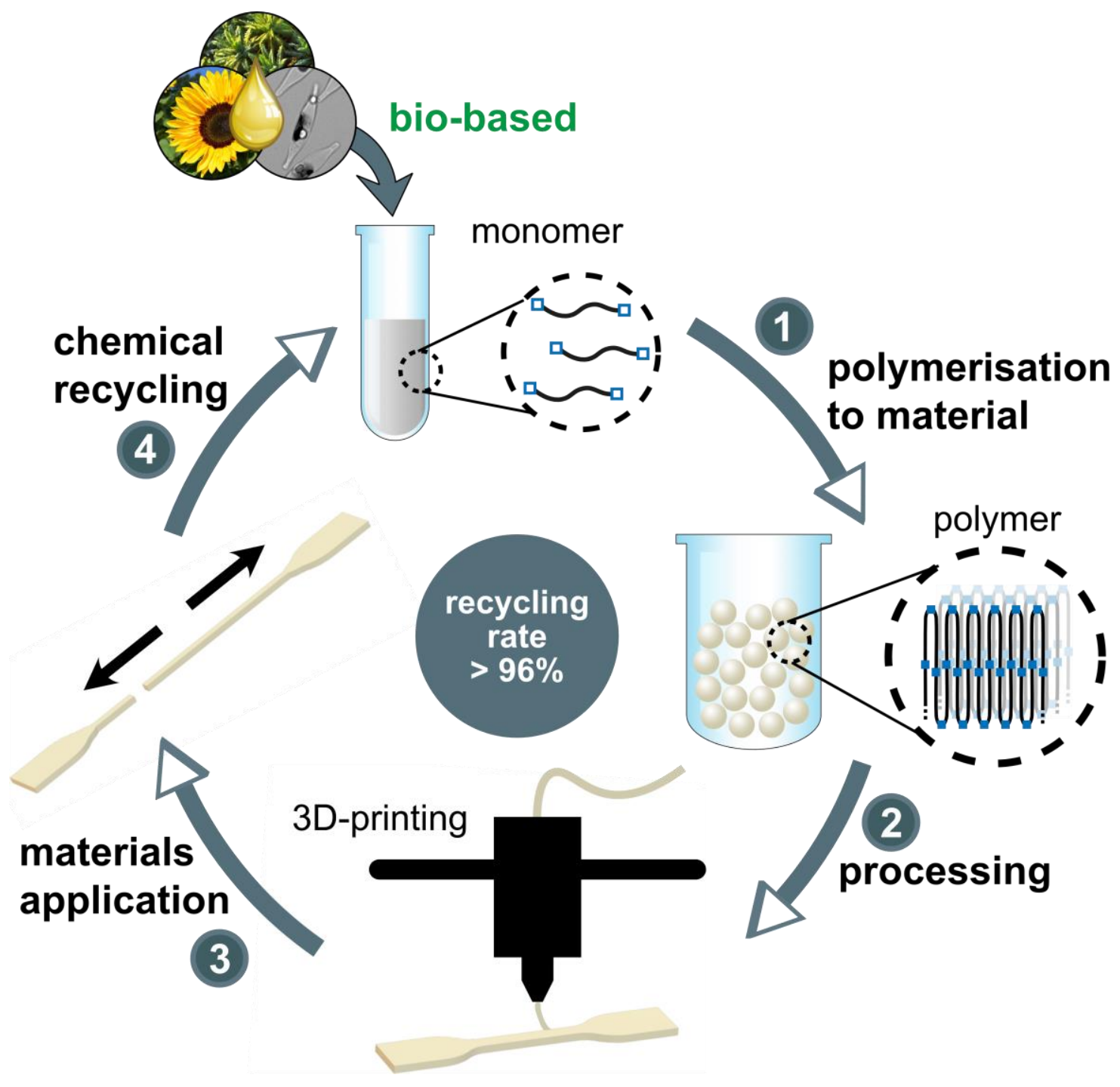

Extended Data Fig. 1 | Closed-loop recycling concept pursued. Polymerisation of long-chain aliphatic building blocks obtained from biorefining of plant or microalgae oils yields polycarbonates and polyesters with polyethylene-

like properties (1). These materials can be processed by common injection moulding and they are also well suited for additive manufacturing (2). After the useful service life of the polyethylene-like polymers - indicated here by a typical specimen for tensile testing - (3), chemical recycling in a solvolysis process to the underlying monomers is enabled by the functional groups present in the polymer backbone (4). The recovered monomers can be repolymerised to materials with properties on par with the initial polymers with an overall recycling rate of $>96 \%$. 
a

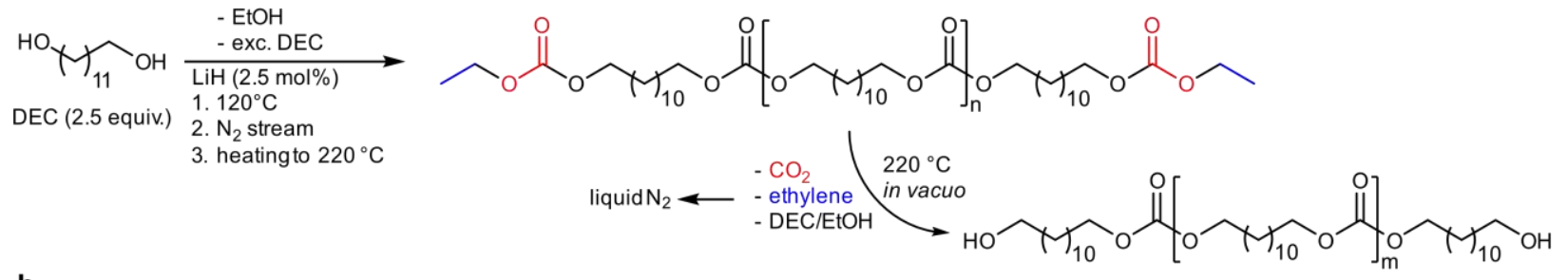

b
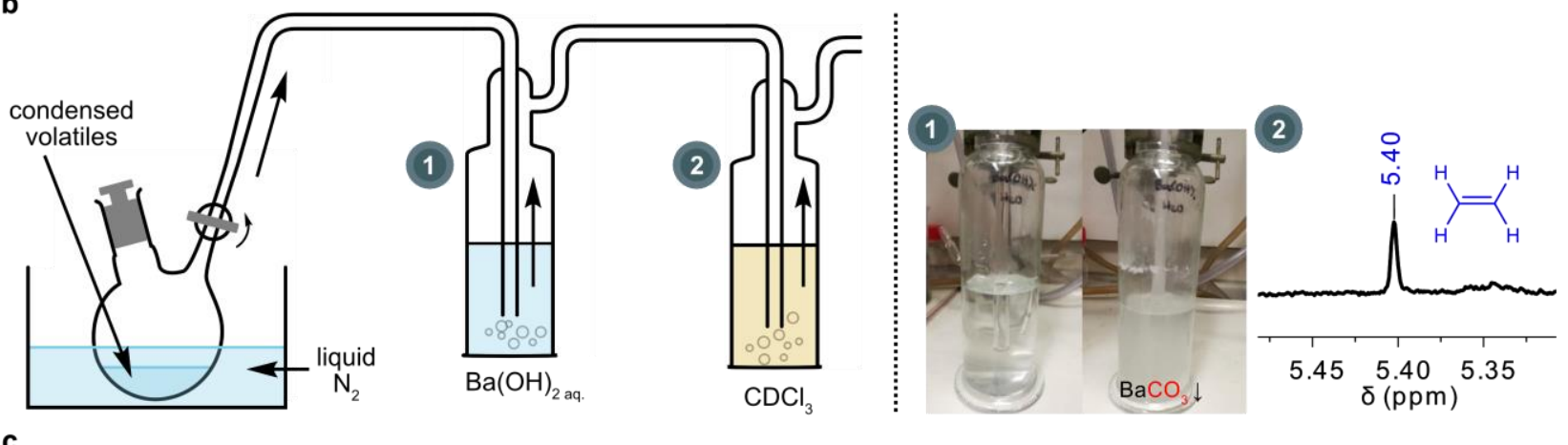

C

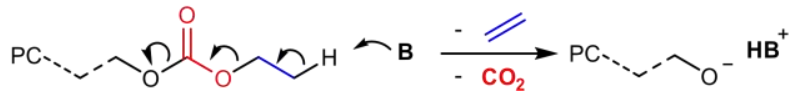

Extended Data Fig. 2 | Decarboxylation of diethyl carbonate endgroups. To achieve high molecular weights for long-chain aliphatic polycarbonates, diethyl carbonate (DEC) was employed as a monomer. Unlike the commonly used dimethyl carbonate ${ }^{19,31}$ this provides an additional pathway for molecular weight build-up. In the final stages of the polycondensation reaction, condensations of two carbonate endgroups with liberation of $\mathrm{ROC}(=\mathrm{O}) \mathrm{OR}$ are the prevailing reactions. This is a kinetically less favored reaction compared to the initially dominant transesterification of carbonate groups with alcohols. This lower reactivity is particularly relevant as in this critical stage of the reaction at high conversions of functional groups the concentration of reacting groups is low. For the case of diethyl carbonate as a monomer, decomposition of $-\mathrm{OC}(=\mathrm{O}) \mathrm{OEt}$ end groups to carbon dioxide and ethylene can yield more reactive $-\mathrm{OH}$ groups. This pathway ${ }^{32}$ enables the decisively higher final product molecular weights observed with diethyl carbonate as a monomer. a, Model reaction of $5 \mathrm{~g}$ of 1,12-dodecanediol with DEC to investigate the decarboxylation of diethyl carbonate endgroups probing for the formation of $\mathrm{CO}_{2}$ and ethylene. $\mathbf{b}$, Schematic experimental setup for the capture of $\mathrm{CO}_{2}$ in aqueous $\mathrm{Ba}(\mathrm{OH})_{2}(1)$ and ethylene in $\mathrm{CDCl}_{3}$ (2) from the condensed volatiles of the model reaction. Photos show the increasing turbidity of the $\mathrm{Ba}(\mathrm{OH})_{2}$ solution upon formation of $\mathrm{BaCO}_{3}(1)$ and a detail of the ${ }^{1} \mathrm{H}-\mathrm{NMR}$ spectrum of the $\mathrm{CDCl}_{3}$ solution with the characteristic resonance for ethylene (2). c, Proposed decomposition mechanism of ethyl carbonate end groups during the polycondensation. B represents a base present in the reaction mixture that can facilitate the abstraction of the terminal proton. 

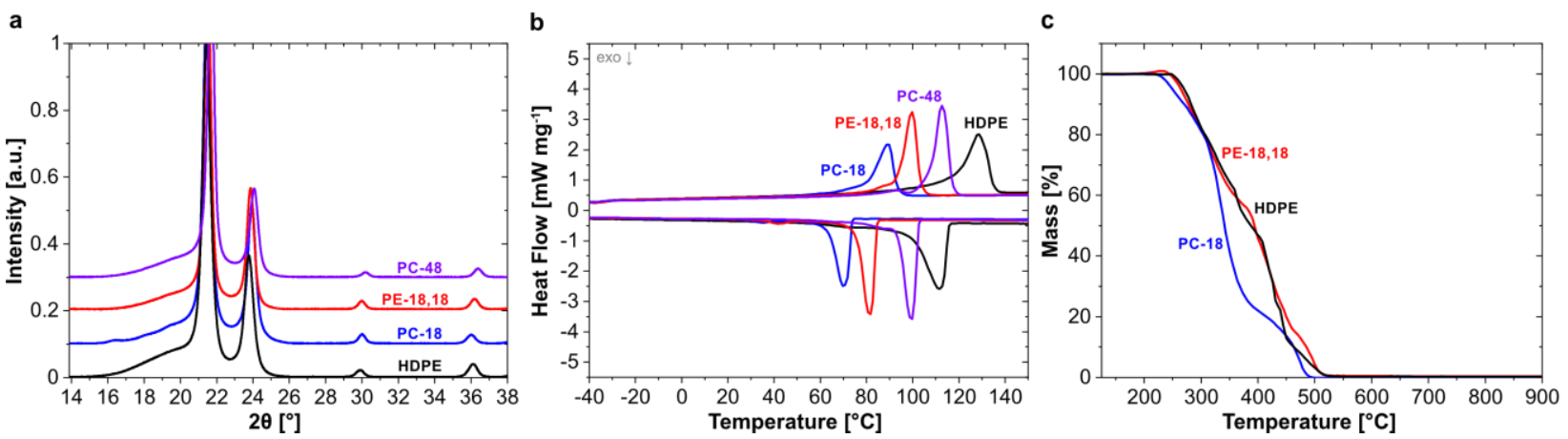

Extended Data Fig. 3 | Thermal and solid-state properties of polymers. a, Comparison of the WAXS diffractograms of PC-18, PE-18,18, PC-48 and HDPE. Reflexes correspond to the orthorhombic unit cell of polyethylene in all cases and all materials exhibit a HDPE-like crystallinity of $\sim 80 \%$. b, Comparison of the DSC traces of PC-18, PE-18,18, PC-48 and HDPE. c, TGA traces of PC-18 and PE-18,18 in comparison to HDPE. 


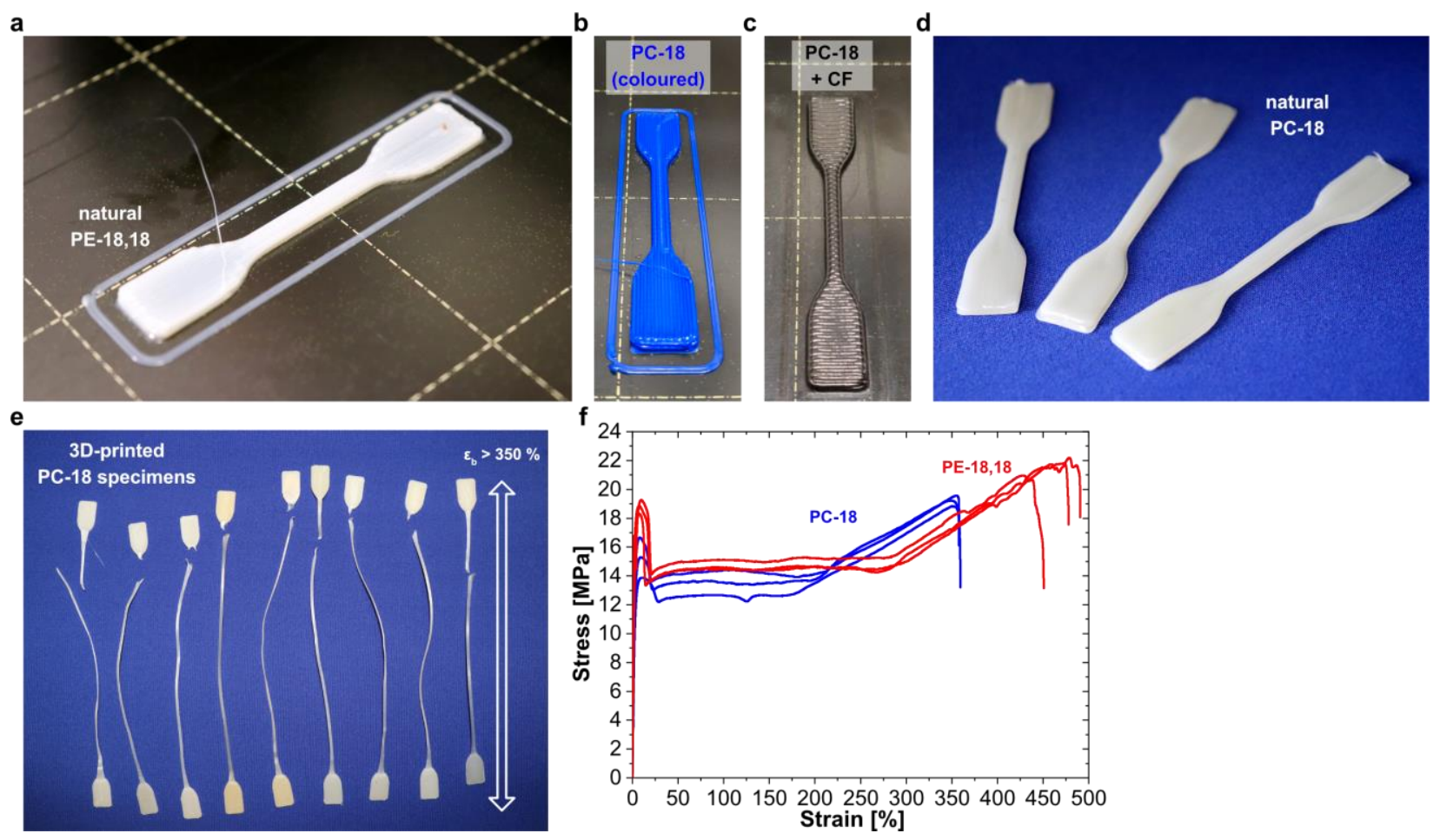

Extended Data Fig. 4 | Mechanical properties of 3D-printed PC-18 and PE-18,18 tensile testing specimens. a, 3D-printed PE-18,18 tensile testing specimen on the print bed. b, 3D-printed blue coloured PC-18 tensile testing specimen on the print bed. c, 3D-printed carbon fibre reinforced PC-18 tensile testing specimen on the print bed. d, 5 3D-printed PC-18 tensile testing specimens after removal from the print bed. e, 3D-printed PC-18 tensile testing specimens after tensile testing. f, Representative stress-strain curves of 3D-printed PC-18 and PE-18,18 tensile testing specimens. 
a

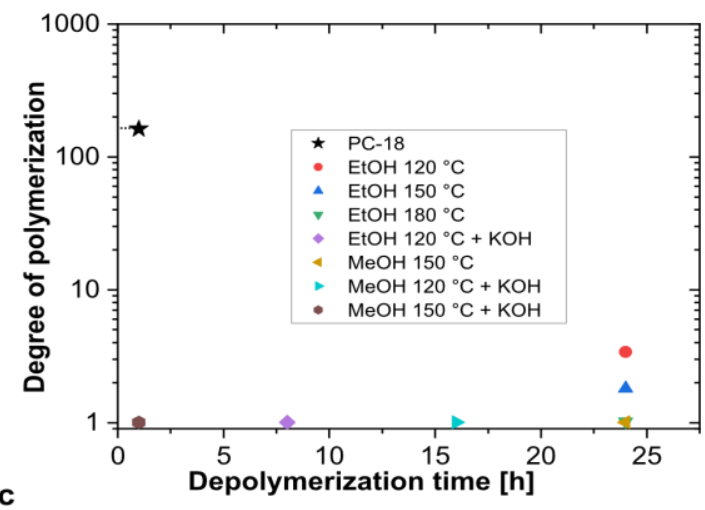

c

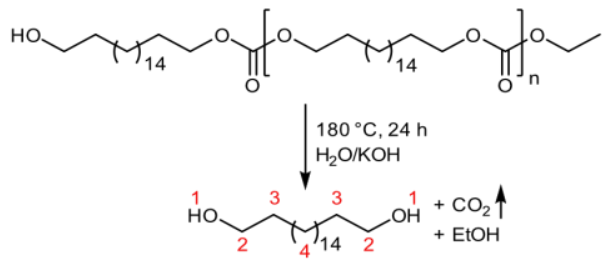

b
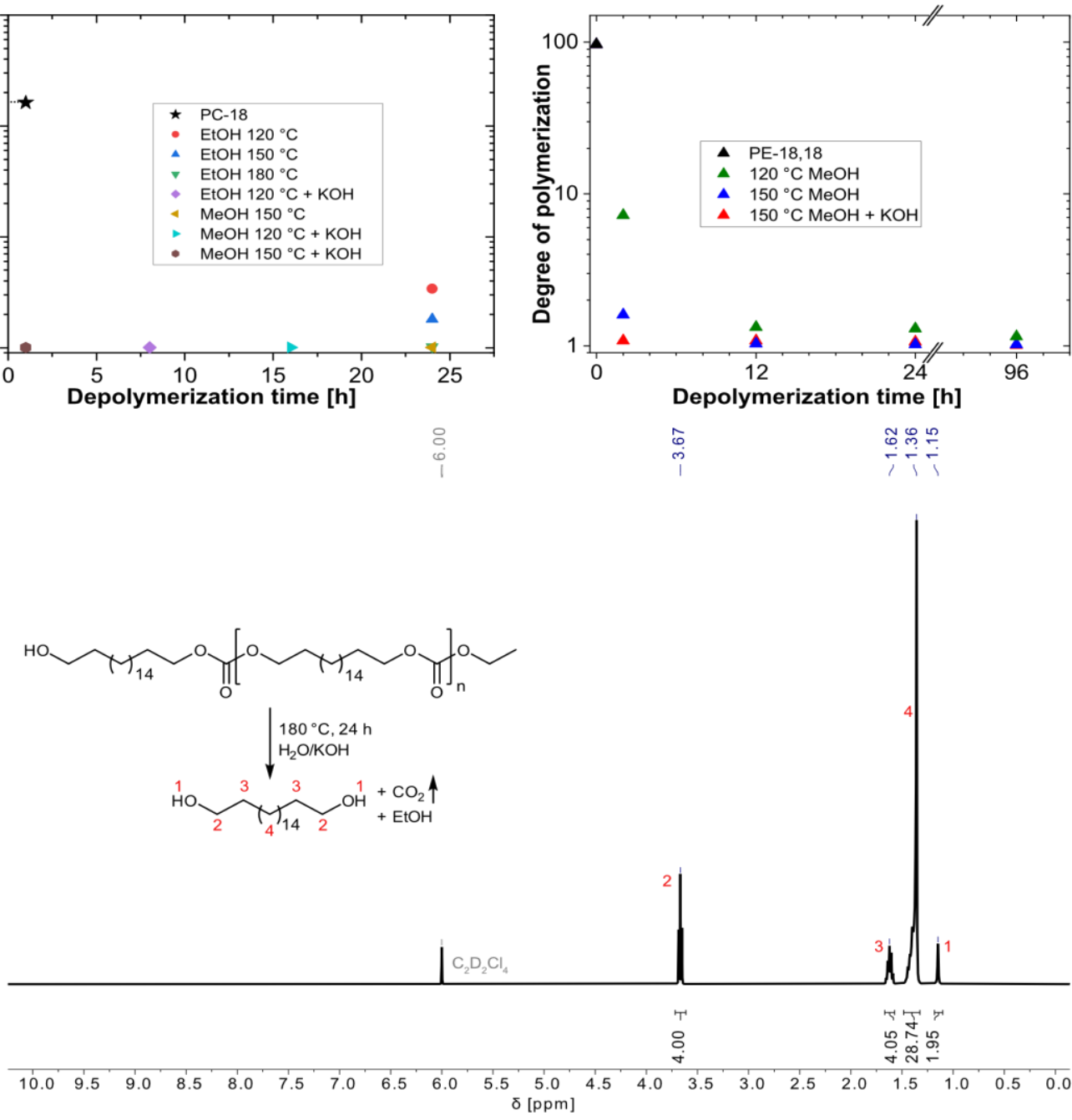

Extended Data Fig. 5 | Depolymerisation of PC-18 and PE-18,18. To assure a facile and rapid depolymerisation in the recycling of the polymers, different conditions were screened for both PC-18 and PE-18,18 in small-scale pressure reactor experiments. $200 \mathrm{mg}$ of polymer were stirred $(500 \mathrm{rpm})$ with $5 \mathrm{~mL}$ of methanol or ethanol, respectively, at the temperature and for the duration listed. Optionally, $10 \mathrm{wt} \% \mathrm{KOH}$ with respect to the mass of polymer were added as a basic depolymerisation catalyst. The volatiles of the obtained reaction mixture were removed under reduced pressure and the degree of polymerisation of the crude product was determined by ${ }^{1} \mathrm{H}-\mathrm{NMR}$ spectroscopy in tetrachloroethane$\mathrm{d}_{2}$ at $383 \mathrm{~K}$. Catalysis of the polyester depolymerisation reaction by $\mathrm{KOH}$ lead to partial saponification of the 1,18dimethyl octadecanedioate monomer. a, Results for PC-18. b, Results for PE-18,18. c, Depolymerisation in water of 10 PC-18 also proceeded completely to the monomer, the carbonate being released as $\mathrm{CO}_{2}$. The ${ }^{1} \mathrm{H}$ NMR spectrum ( $383 \mathrm{~K}$, $400 \mathrm{MHz}, \mathrm{C}_{2} \mathrm{D}_{2} \mathrm{Cl}_{4}$ ) shows the precipitated hydrolysis product 1,18-octadecane diol obtained by filtration of the reaction mixture. 
a

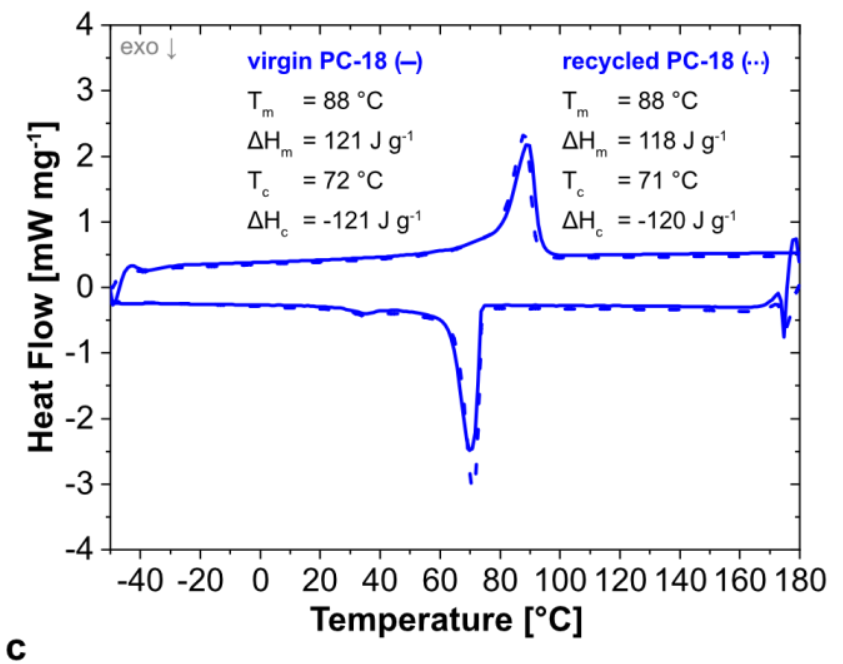

C

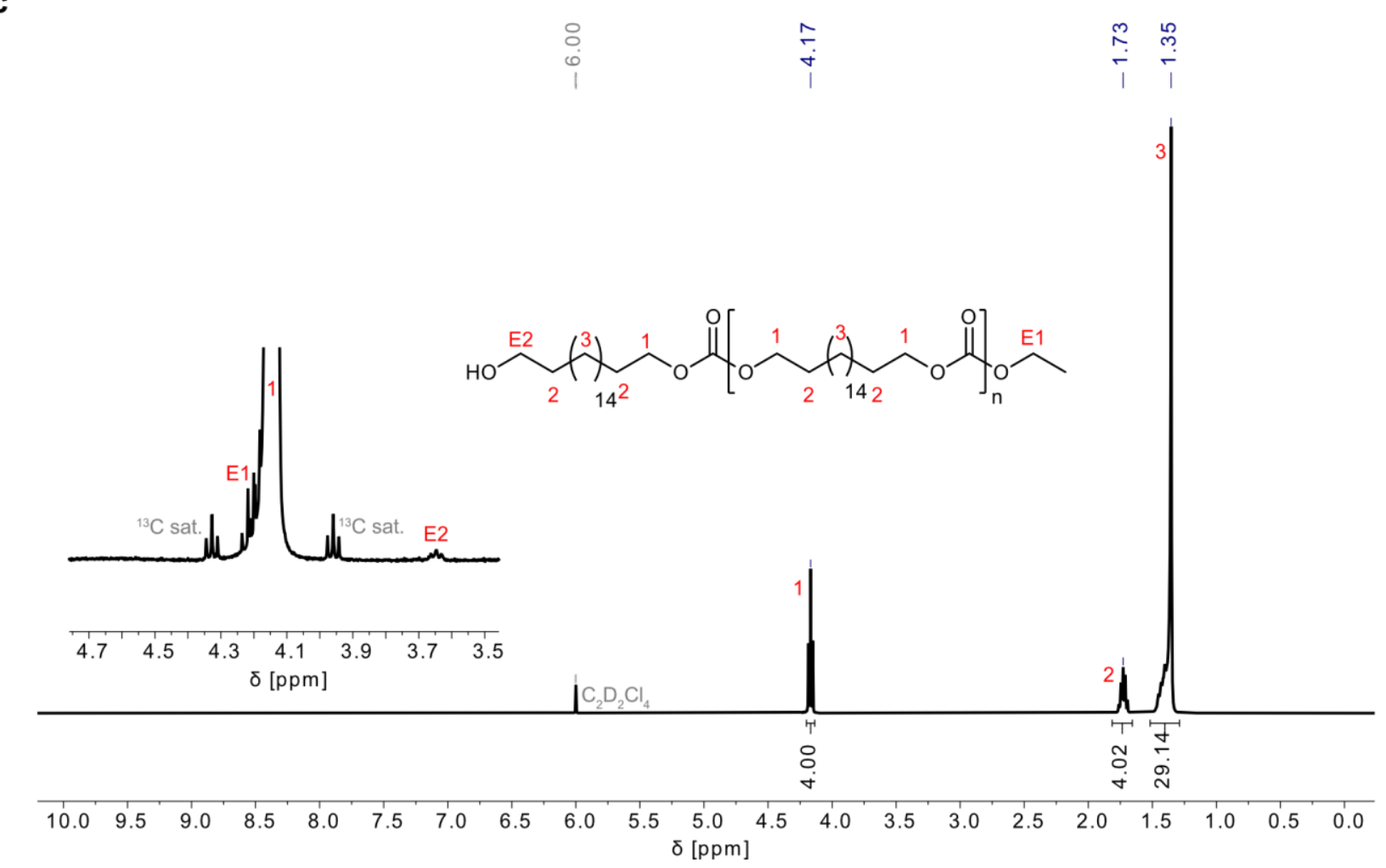

b

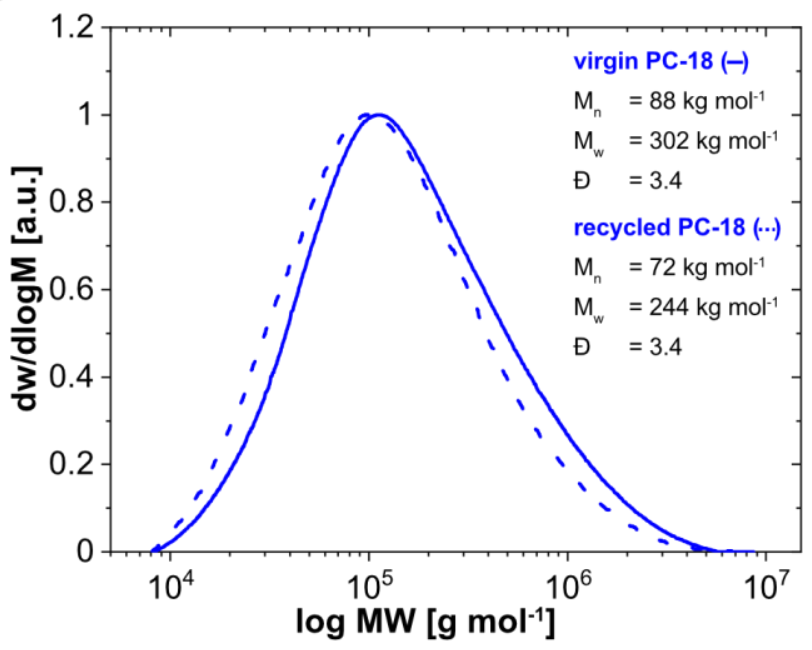



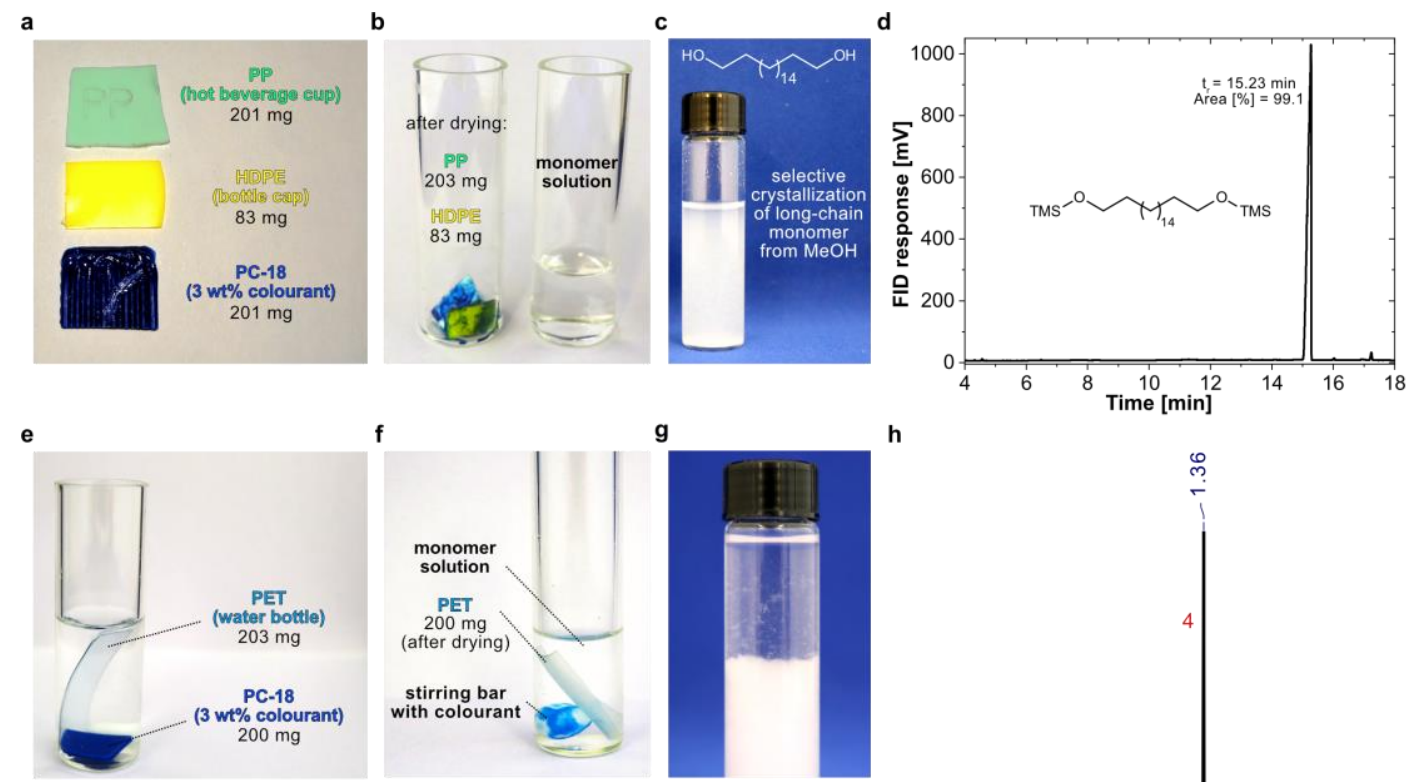

f
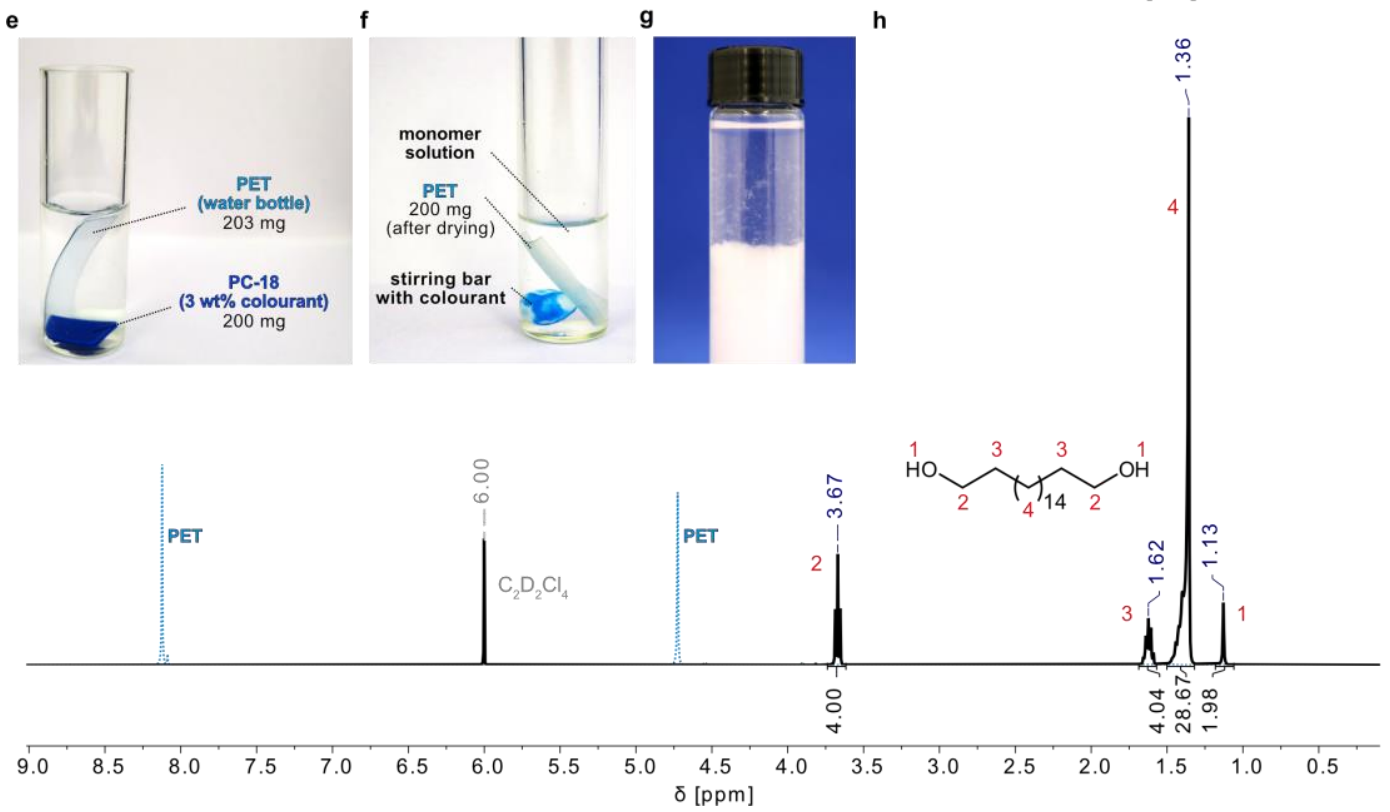

Extended Data Fig. 7 | Depolymerisation of PC-18 in presence of polyolefins and PET. To simulate a potential waste stream, methanolysis of coloured PC-18 was conducted in the presence of polyolefin samples (HDPE, PP) and PET, respectively. a, Blue coloured PC-18 and specimens of HDPE and PP with their respective weights before depolymerisation. b, In contrast to the depolymerised and dissolved PC-18 (right reactor inlet), the polyolefin samples remained unchanged during the solvolysis process and could be separated from the monomer solution formed from PC-18, with the blue colourant deposited on their surfaces (left reactor inlet). c, 1,18-octadecane diol crystallised from the $\mathrm{MeOH}$ monomer solution. d, GC analysis of 1,18-octadecane diol recovered. e, Specimens of blue coloured PC18 and PET with their respective weights before depolymerisation. f, Depolymerisation mixture after $24 \mathrm{~h}$ at $150{ }^{\circ} \mathrm{C}$ in $\mathrm{MeOH}$. The PC-18 specimen depolymerised to a monomer solution while the PET specimen retained its shape and could be separated. The blue colourant was deposited on the surface of the stirring bar. g, 1,18-Octadecane diol crystallised from the $\mathrm{MeOH}$ monomer solution. $\mathbf{h},{ }^{1} \mathrm{H}$ NMR spectrum $\left(383 \mathrm{~K}, 400 \mathrm{MHz}, \mathrm{C}_{2} \mathrm{D}_{2} \mathrm{Cl}_{4}\right)$ of 1,18-octadecane diol recovered by depolymerisation in presence of PET. The characteristic resonances for PET (dashed blue spectrum, shown for comparison) underlining the successful separation. 

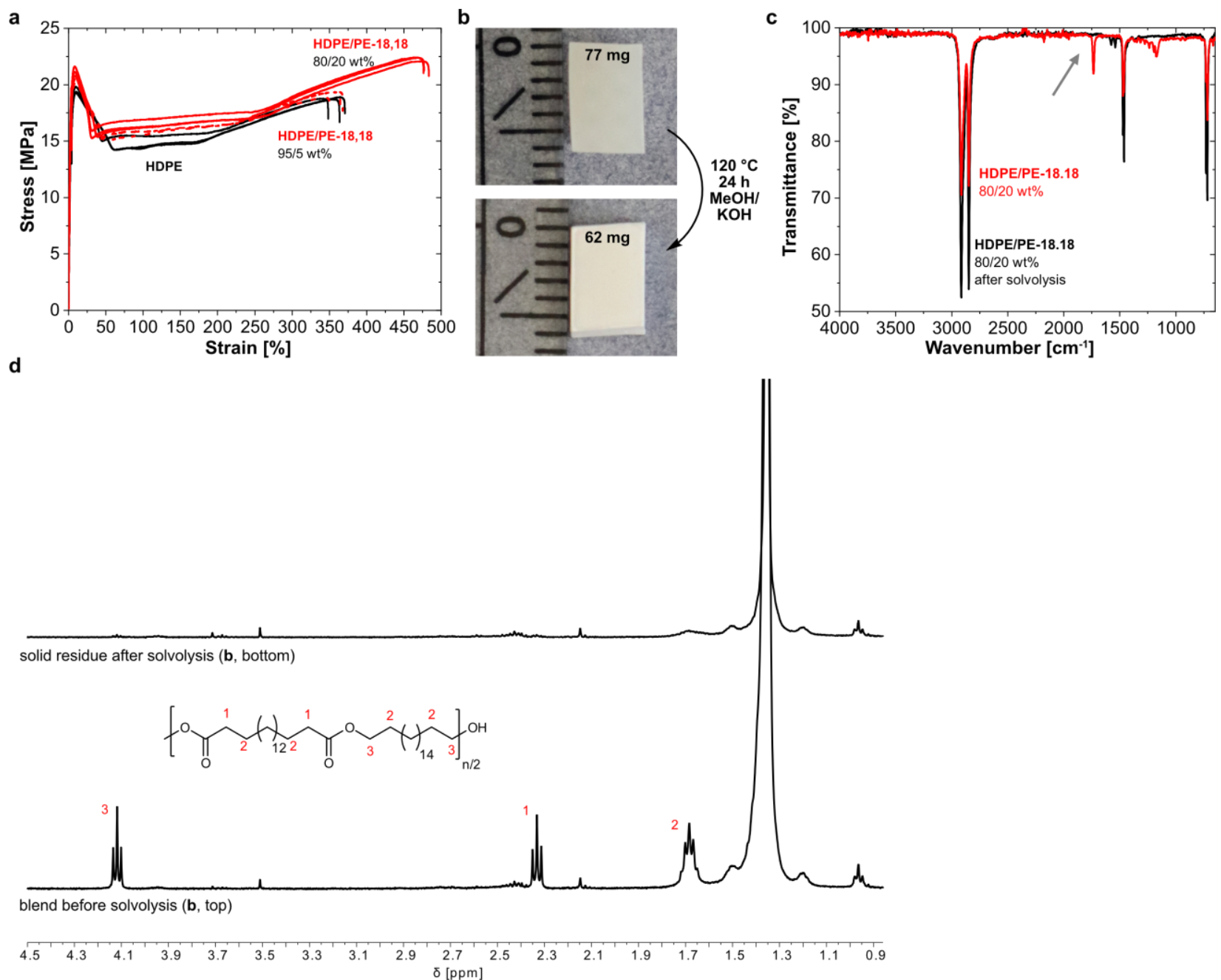

Extended Data Fig. 8 | Depolymerisation of PE-18,18 from a blend with HDPE. To simulate a potential contamination of an HDPE waste stream, blends of HDPE with PE-18,18 were generated by melt compounding. a, Tensile properties of an 80/20 wt $\%$ and 95/5 wt $\%$ HDPE/PE-18,18 blend. These show no adverse impact of the presence of PE-18,18 compared to the neat HDPE. b, Photo of a HDPE/PE-18,18 80/20 wt $\%$ blend specimen before and after depolymerization by methanolysis $\left(120{ }^{\circ} \mathrm{C}, 10 \mathrm{wt} \% \mathrm{KOH}, 24 \mathrm{~h}\right)$. c, IR spectra and d, ${ }^{1} \mathrm{H}-\mathrm{NMR}$ spectra $\left(\mathrm{C}_{2} \mathrm{D}_{2} \mathrm{Cl}_{4}, 383 \mathrm{~K}, 400 \mathrm{MHz}\right)$ of the original HDPE/PE-18,18 80/20 wt\% blend and of the residue from depolymerisation. The absence of the carbonyl band and of the characteristic ester ${ }^{1} \mathrm{H}$ resonances demonstrates the removal of the PE-18,18 component from the blend. 
a

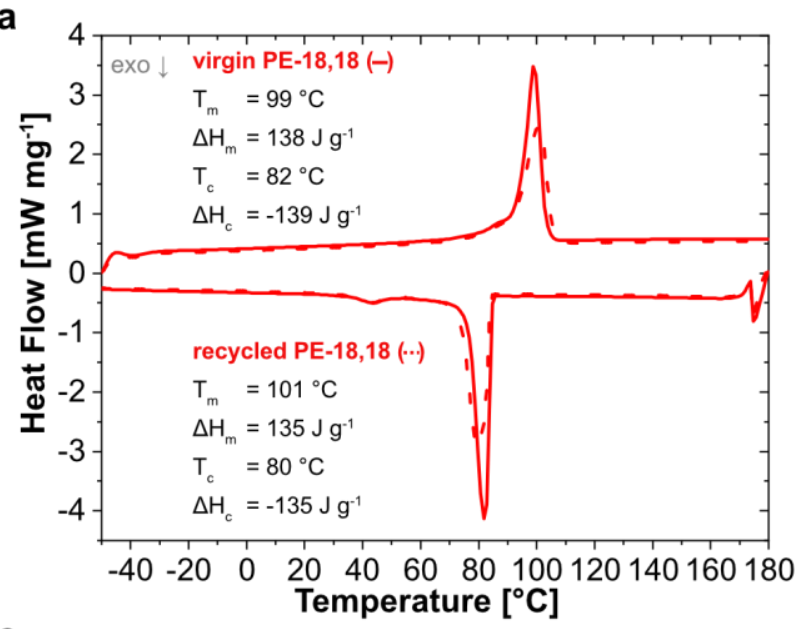

C
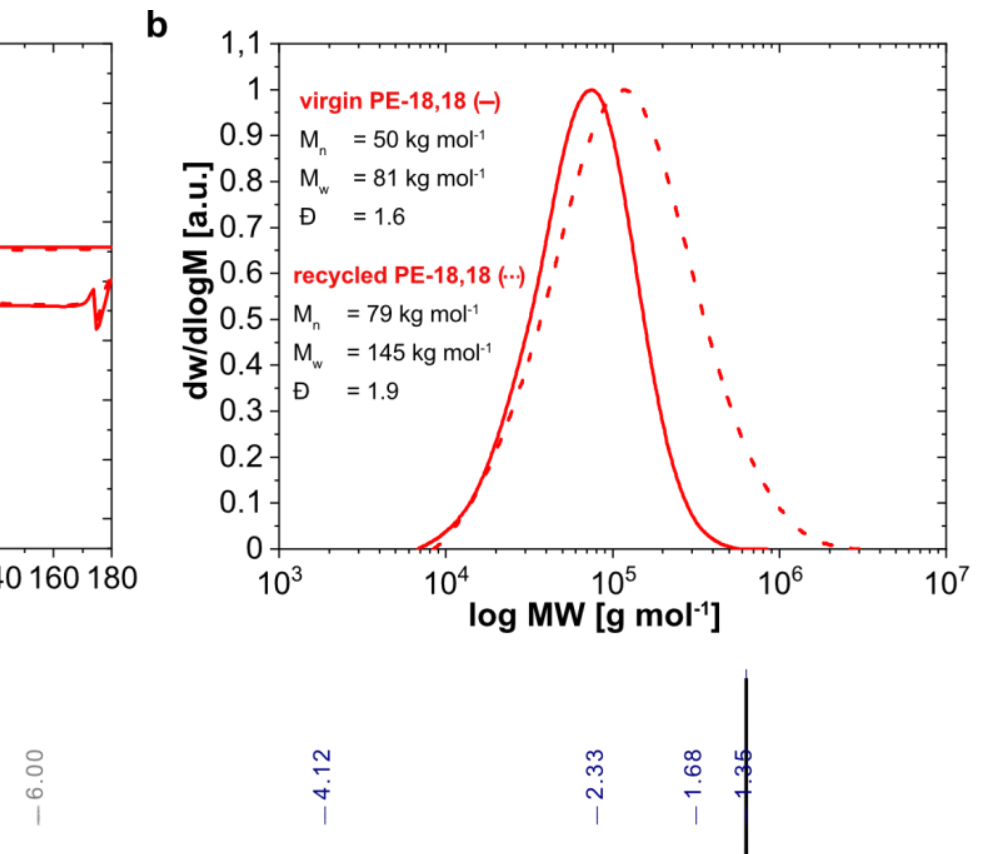
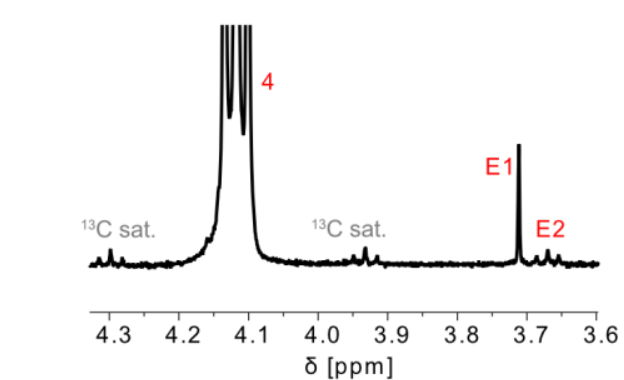<smiles>[R20]C(C)(O)CC(C)(C)CCOC(=O)CCC(C)(C)CCC(=O)OC(C)(C)C</smiles><smiles>CCCCCCO</smiles><smiles>CCOC(C)=O</smiles>

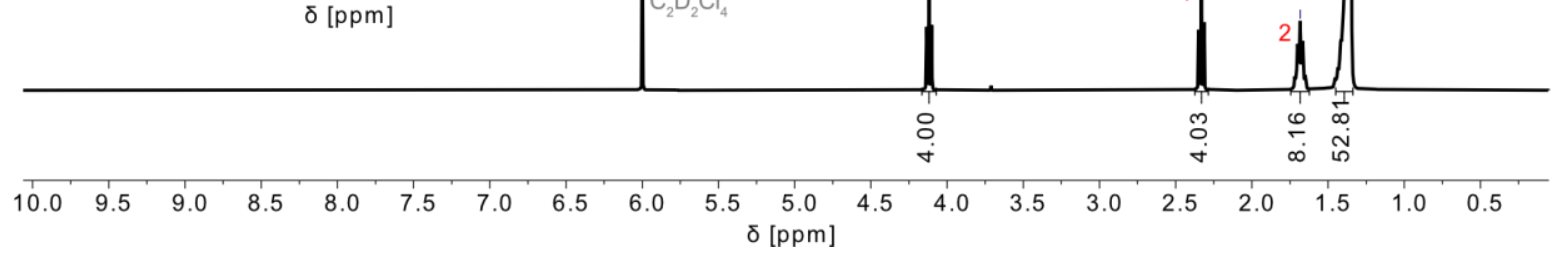

Extended Data Fig. 9 | Properties of recycled PE-18,18. a, DSC traces of virgin and recycled PE-18,18. b, GPC traces of virgin and recycled PE-18,18. c, ${ }^{1} \mathrm{H}-\mathrm{NMR}$ spectrum $\left(\mathrm{C}_{2} \mathrm{D}_{2} \mathrm{Cl}_{4}, 383 \mathrm{~K}, 400 \mathrm{MHz}\right)$ of recycled PE-18,18. 